\title{
Three-dimensional Voronoï imaging methods for the measurement of near-wall particulate flows
}

\author{
B. Spinewine, H. Capart, M. Larcher, Y. Zech
}

\begin{abstract}
A set of stereoscopic imaging techniques is proposed for the measurement of rapidly flowing dispersions of opaque particles observed near a transparent wall. The methods exploit projective geometry and the Voronoï diagram. They rely on purely geometrical principles to reconstruct $3 \mathrm{D}$ particle positions, concentrations, and velocities. The methods are able to handle position and motion ambiguities, as well as particle-occlusion effects, difficulties that are common in the case of dense dispersions of many identical particles. Fluidization cell experiments allow validation of the concentration estimates. A mature debris-flow experimental run is then chosen to test the particle-tracking algorithm. The Voronoï stereo methods are found to perform well in both cases, and to present significant advantages over monocular imaging measurements.
\end{abstract}

\section{1}

\section{Introduction}

Flows of disperse phases are involved in a wide variety of situations of scientific and engineering interest. These include liquid-entrained gas bubbles, aerosols, dry granular flows, fluidized beds of particles, and liquid-saturated

Received: 8 August 2002 / Accepted: 12 September 2002

Published online: 19 December 2002

(C) Springer-Verlag 2002

\section{B. Spinewine $(\square)$}

Fonds pour la Recherche dans l'Industrie et l'Agriculture and Department of Civil and Environmental Engineering,

Université catholique de Louvain, place du Levant 1 ,

1348 Louvain-la-Neuve, Belgium

E-mail: spinewine@gce.ucl.ac.be

Tel.: +32-10-472123

Fax: $+32-10-472179$

H. Capart

Department of Civil Engineering,

National Taiwan University,

Taipei 10617, Taiwan

M. Larcher

CUDAM - Dipartimento di Ingegneria Civile ed Ambientale, Università degli Studi di Trento,

Via Mesiano 77, 38050 Trento,

Italy

Y. Zech

Department of Civil and Environmental Engineering,

Université catholique de Louvain, place du Levant 1 ,

1348 Louvain-la-Neuve,

Belgium particulate currents. In many of these situations, the individual elements (bubbles or grains) can be approximated as rigid bodies undergoing distinct motions, and the dynamic system can be abstracted into an evolving configuration of particle positions (Campbell 1990; Zhang and Prosperetti 1994; Kang et al. 1997). The system behavior in the dense limit is often of particular interest, featuring active particles interacting with each other, and adopting flow-induced preferential arrangements (Fortes et al. 1987; Savage and Dai 1993; Rouyer et al. 2000). The present work addresses some of the experimental challenges associated with such flows through the development of novel stereo imaging techniques.

We consider the following typical measurement setup (Fig. 1). The flow of interest involves a dense ensemble of identical opaque particles immersed in a transparent fluid and imaged by twin cameras through a transparent plane (e.g., flume side-wall). Because of the high concentration of particles, optical penetration within the bulk is limited to a depth of a few grain diameters. Measurements sought include the 3D positions of the visible grains, the in-plane (i.e., parallel to the image plane), and out-ofplane (normal to the image plane) particle velocities, and the volumetric particle concentration. Two main problems must be faced in deriving such measurements: (1) particle-pairing ambiguities, which hamper both stereoscopic matching and velocimetric tracking; this is especially true for dense, rapidly sheared dispersions of identical particles undergoing irregular motions; (2) particle occlusion, which biases estimates of the volumetric particle concentration; in the dense case, the apparent concentration of visible particles is not equivalent to the actual concentration of physical particles.

In some instances, it is possible to circumvent these difficulties. Problems caused by matching and tracking ambiguities can be diminished by focusing only on the positions and motions of a subset of marked particles or tracers. Problems caused by occlusion, on the other hand, can be bypassed by using refractive index matching techniques (Cui and Adrian 1997) or full volumetric scanning as in nuclear magnetic resonance imaging (Phillips et al. 1992; Nakagawa et al. 1993; Seymour et al. 2000). A number of other non-intrusive methods have been used for the dynamic characterization of opaque solid-liquid flows, including positron emission particle tracking (Fairhurst et al. 2001; Wildman et al. 2001), and diffusing wave spectroscopy (Menon and Durian 1997). These techniques, however, are not always feasible and present their own drawbacks. The use of subsets of marked 


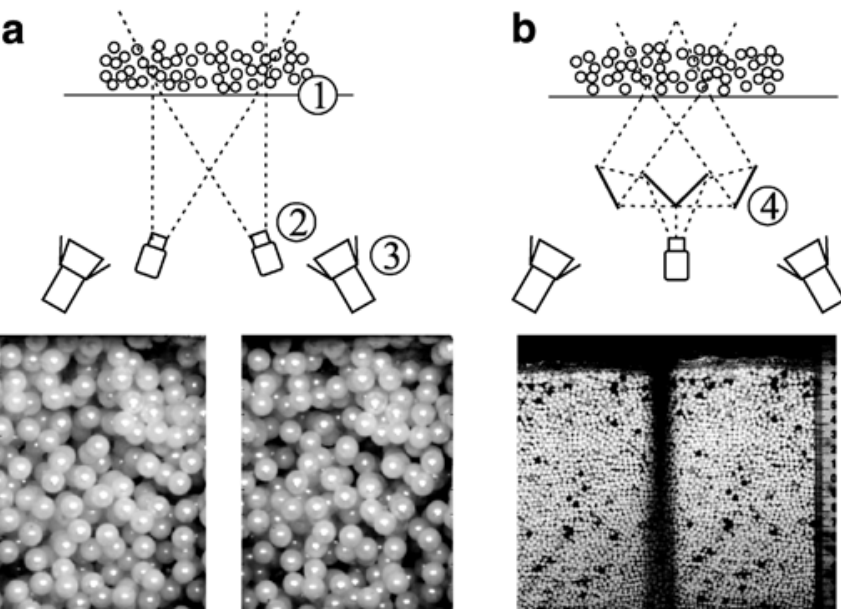

Fig. 1a, b. Stereoscopic imaging of near-wall dispersion of particles: a using two synchronized sensors; $b$ using a single sensor with system of mirrors. (1) transparent side-wall; (2) CCD camera; (3) light source; (4) mirrors

particles, as in PEPT for example, can yield good Lagrangian statistics for the motion, but misses the spatial correlation and particle arrangement effects that act on the scale of a few particle diameters. Refractive index matching, in addition, is only possible for liquid-solid mixtures of very special optical properties. The approach of the present paper is to stick with more widely available test materials and digital cameras, and to deal with the ambiguity and occlusion problems in the context of stereo imaging methods.

Stereo imaging techniques applied to dilute dispersions (passive tracers seeding a fluid flow) have been described in the review of Adrian (1991) and in the studies of Malik et al. (1993), Ushijima and Tanaka (1996), and Virant and Dracos (1997). A method to obtain concentration estimates from statistical distributions associated with stereo imaging techniques is described in Murai et al. (2001). Monocular imaging methods, on the other hand, have been applied to dense flows by various experimenters. Most of these works have been restricted to $2 \mathrm{D}$ analogues, e.g., a monolayer of disks or spheres sandwiched between two parallel plates (Drake 1991; Azanza et al. 1999; Wildman et al. 1999), or to the imaging of a subset of marked particles (Natarajan et al. 1995; Hryciw et al. 1997; Hsiau and Jang 1998). In Capart et al. (2002), monocular imaging methods were proposed for the measurement of the discrete kinematics of full sets of visible grains in a dense, rapidly sheared dispersion imaged near the sidewall. The issues associated with stereo imaging of dense 3D dispersions do not seem to have been specifically addressed before.

The approach adopted follows the work of Capart (2000) and Capart et al. (2002). The core of the proposed methods consists in exploiting the special properties of Voronoï diagrams (Ahuja 1982; Okabe et al. 1992). These define spatial tessellations of the 2D plane or 3D space into cells centered around individual feature points (see Sect. 3). After abstraction of digital images into discrete sets of point-like particle positions, the Voronoï diagram is used for the three main steps of the analysis: (1) stereoscopic matching of particles; (2) estimation of volumetric concentration; (3) pattern-based particle tracking. Presentation of these various methods constitutes the central section of the present paper. This is preceded by an outline of the 2D particle-identification technique and generic $3 \mathrm{D}$ ray tracing concepts, which are needed as basic tools. The final part of the paper is devoted to the application of the methods to two particulate flows in water. The first case, a homogeneous fluidized bed of light particles, is of particular interest for the validation of concentration estimation methods. The second case is a mature debris flow of PVC particles featuring steep concentration and velocity gradients, and constitutes a challenging test for the particle-tracking techniques. Monocular imaging results for similar flows were presented in Capart et al. (2002), while some preliminary stereo results were described in Douxchamps et al. (2000) and Spinewine et al. (2000).

2

\section{Particle identification and ray geometry}

This first section outlines the basic methods required to pinpoint particles on 2D images and to reconstruct their positions in 3D space. While these basic methods are not new, their presentation sets the stage for the more original Voronoï developments of the next section. The following notations are adopted throughout the paper: upper-case letters denote 2D imaging quantities measured in pixels and ticks (intervals of time separating successive images); lower-case letters denote 3D world quantities measured in meters and seconds. In both cases, vectors are indicated in bold face.

\section{1}

\section{Particle identification}

The first step of the analysis consists in the localization of particle centroids on individual images. For each instant at which synchronized images are acquired under two viewpoints $A$ and $B$, one seeks to identify sets of particle positions $\left\{\mathbf{R}_{i}^{(\mathrm{A})}\right\}$ and $\left\{\mathbf{R}_{j}^{(\mathrm{B})}\right\}$ on the two digital frames. Particle images show up as white blobs of a certain size against a dark background (see Fig. 2a). An image neighborhood associated with a particle can be approximated by a Gaussian gray-level function centered on the particle centroid, with a diameter $D$ that scales with the pixel diameter of the particle. Such bell-like regions are highlighted by convoluting the image with a Laplacian-ofGaussian (Mexican hat) filter of width $D$ (Jähne 1995), as shown in Fig. $2 b$ and c. Local brightness maxima of the highlighted images are then identified iteratively using a "dish-clearing" algorithm: a global maximum is found, then a Gaussian bell of diameter $D$ is subtracted from the neighborhood gray-level values; a new global maximum is found, and so on. The position of each maximum is finally refined to subpixel accuracy by fitting a second-degree interpolation surface around the discrete pixel position. The resulting set of particle centers is shown in Fig. 2 d. The expected rms accuracy on the $\mathrm{X}$ and $\mathrm{Y}$ image coordinates obtained in this fashion is of the order of 0.25 pixel 

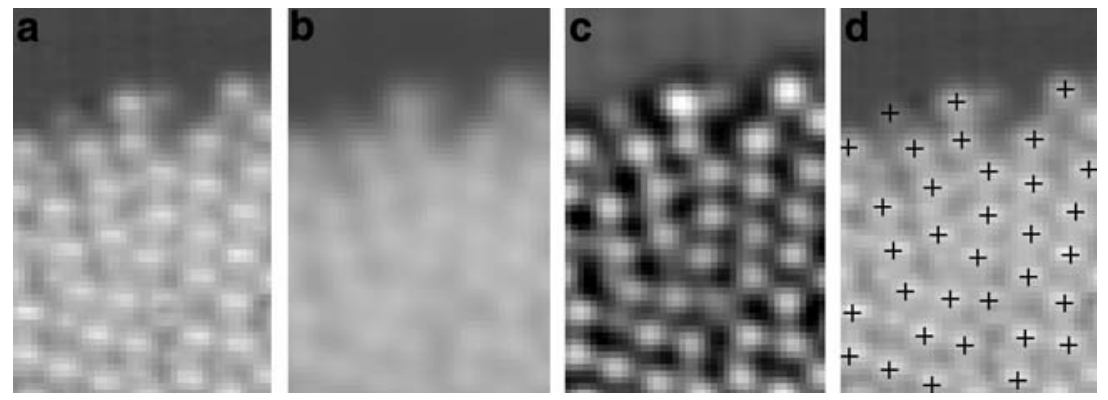

Fig. 2a-d. Particle-identification procedure: $\mathbf{a}$ image fragment; $\mathbf{b}$ image $\mathbf{a}$ after low pass filtering; $\mathbf{c}$ image $\mathbf{b}$ after high pass filtering; d particle positions at brightness maxima of $\mathbf{c}$

(Veber et al. 1997). More details about the procedure are given in Capart et al. (2002).

\section{2}

\section{Transformation between world and camera coordinates}

A transformation is now required to relate the set of $2 \mathrm{D}$ image coordinates of any point $\mathrm{P}$ to its world coordinates. Define $\mathbf{r}_{\mathrm{P}}=\left[\begin{array}{lll}x_{\mathrm{P}} & y_{\mathrm{P}} & z_{\mathrm{P}}\end{array}\right]^{\mathrm{T}}$ as the world coordinates of point $\mathrm{P}$ (Fig. 3), and $\mathbf{R}_{\mathrm{P}}^{(\mathrm{A})}=\left[X_{\mathrm{P}}^{(\mathrm{A})} Y_{\mathrm{P}}^{(\mathrm{A})}\right]^{\mathrm{T}}$ as the $2 \mathrm{D}$ image coordinates of point $P$ associated with the camera viewpoint $A$ (point $\mathrm{P}^{\prime}$ in left image plane $\Phi$ ). The transformation is obtained by modeling the image formation as a central projection from a virtual camera focal point $A$ onto the image plane $\Phi$ (Tsai 1987; Jain et al. 1995). Conserving the alignment of points, this projective transformation constitutes a reasonable approximation of the image formation process and facilitates tremendously the ray tracing and matching operations (Trucco and Verri 1998; Faugeras 1999). For each viewpoint A, one can then specify a matrix $\left[\mathrm{A}^{(\mathrm{A})}\right]$ and a vector $\mathbf{b}^{(\mathrm{A})}$ such that

$\alpha\left[\begin{array}{c}X^{(\mathrm{A})} \\ Y^{(\mathrm{A})} \\ 1\end{array}\right]=\left[\mathbf{A}^{(\mathrm{A})}\right]\left[\begin{array}{l}x \\ y \\ z\end{array}\right]+\mathbf{b}^{(\mathrm{A})}$

where $\alpha$ is a scalar parameter best interpreted in the context of Eq. (5) below. Matrix $\left[\mathbf{A}^{(\mathrm{A})}\right]$ and vector $\mathbf{b}^{(\mathrm{A})}$, on

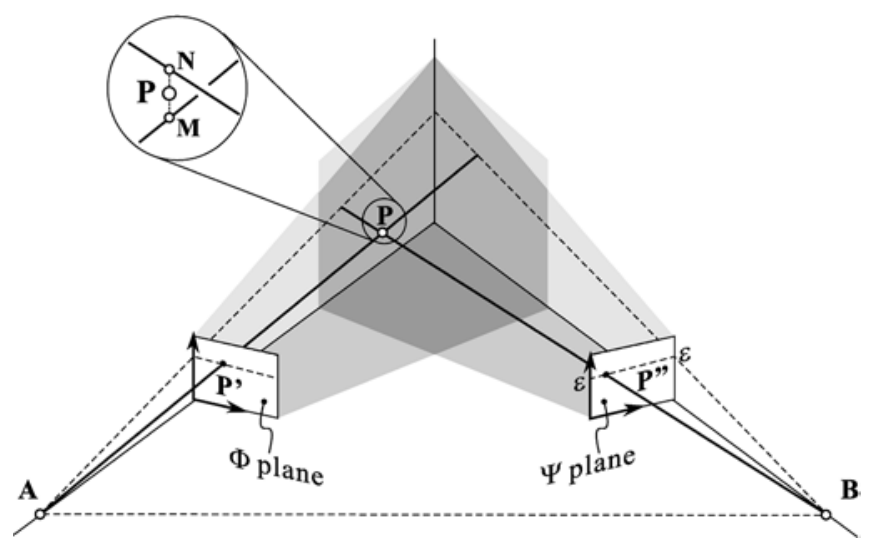

Fig. 3. Imaging geometry: physical point $\mathrm{P}$ and its image projections $\mathrm{P}^{\prime}$ and $\mathrm{P}^{\prime \prime}$ on the two stereo views. Rays emanate from focal points $A$ and $B$ of the two central projections. The trace of the epipolar plane comprising P is shown in dashed lines. Inset: due to imperfections in the imaging geometry, the two rays may not perfectly intersect the other hand, must be calibrated from a set of points $\mathrm{P}_{k}$ for which both the world coordinates $\left[x_{\mathrm{k}} y_{\mathrm{k}} z_{\mathrm{k}}\right]^{\mathrm{T}}$ and the image coordinates $\left[X_{k}^{(\mathrm{A})} Y_{k}^{(\mathrm{A})}\right]^{\mathrm{T}}$ are known. Eliminating parameter $\alpha$ from (1), one obtains for each calibration point $\mathrm{P}_{k}$ two linear equations in the unknown coefficients $a_{i j}^{(\mathrm{A})}$ and $b_{j}^{(\mathrm{A})}$ of $\left[\mathrm{A}^{(\mathrm{A})}\right]$ and $\mathbf{b}^{(\mathrm{A})}$ :

$$
\begin{aligned}
& x_{k} a_{11}^{(\mathrm{A})}+y_{k} a_{12}^{(\mathrm{A})}+z_{k} a_{13}^{(\mathrm{A})}-x_{k} X_{k}^{(\mathrm{A})} a_{31}^{(\mathrm{A})}-y_{k} X_{k}^{(\mathrm{A})} a_{32}^{(\mathrm{A})} \\
& \quad-z_{k} X_{k}^{(\mathrm{A})} a_{33}^{(\mathrm{A})}+b_{1}^{(\mathrm{A})}-X_{k}^{(\mathrm{A})} b_{3}^{(\mathrm{A})}=0, \\
& x_{k} a_{21}^{(\mathrm{A})}+y_{k} a_{22}^{(\mathrm{A})}+z_{k} a_{23}^{(\mathrm{A})}-x_{k} Y_{k}^{(\mathrm{A})} a_{31}^{(\mathrm{A})}-y_{k} Y_{k}^{(\mathrm{A})} a_{32}^{(\mathrm{A})} \\
& \quad-z_{k} Y_{k}^{(\mathrm{A})} a_{33}^{(\mathrm{A})}+b_{2}^{(\mathrm{A})}-Y_{k}^{(\mathrm{A})} b_{3}^{(\mathrm{A})}=0 .
\end{aligned}
$$

A minimum of six calibration points $\mathrm{P}_{k}$ (12 equations) are thus required to determine the 12 unknown coefficients. Since the system is homogeneous, one needs to append one more equation, e.g.,

$b_{1}^{(\mathrm{A})}=1$,

to avoid the trivial solution with all zero coefficients.

The system is further over-determined if more than six calibration points are used, hence a least-square procedure is needed to obtain an optimal solution. In practice, it is recommended to use a large number of calibration points with positions distributed evenly inside the viewing volume.

Once calibrated, Eq. (1) can be used to obtain image coordinates from known world coordinates. Conversely, a point $\mathrm{P}$ having its projection $\mathrm{P}^{\prime}$ with image coordinates $\mathbf{R}_{\mathrm{P}}^{(\mathrm{A})}=\left[X_{\mathrm{P}}^{(\mathrm{A})} Y_{\mathrm{P}}^{(\mathrm{A})}\right]^{\mathrm{T}}$ under viewpoint $\mathrm{A}$ is known to belong to a ray $\mathrm{AP}$ (or $\mathrm{AP}^{\prime}$ as seen in Fig. 3) defined by parametric equation

$\mathbf{r}_{\mathrm{AP}}(\alpha)=\mathbf{r}_{\mathrm{A}}+\alpha \mathbf{s}_{\mathrm{AP}}$,

where $\alpha$ is a free parameter, and vectors $\mathbf{r}_{\mathrm{A}}$ and $\mathbf{s}_{\mathrm{AP}}$ are given by

$\mathbf{r}_{\mathrm{A}}=-\left[\mathbf{A}^{(\mathrm{A})}\right]^{-1} \mathbf{b}^{(\mathrm{A})}$,

$\mathbf{s}_{\mathrm{AP}}=\left[\mathbf{A}^{(\mathrm{A})}\right]^{-1}\left[\begin{array}{c}X_{\mathrm{P}}^{(\mathrm{A})} \\ Y_{\mathrm{P}}^{(\mathrm{A})} \\ 1\end{array}\right]$. 
The vector $\mathbf{r}_{\mathrm{A}}$ is the position of the focal point $\mathrm{A}$ of the projection, and lies at the intersection of all rays associated with viewpoint $A$. It is obtained by setting $\alpha$ to zero in (1). The vector $\mathbf{s}_{\mathrm{AP}}$, on the other hand, specifies the direction of the particular ray which pierces the image plane at $\mathrm{P}^{\prime}$. It is obtained as the difference $\mathbf{r}_{\mathrm{P}}-\mathbf{r}_{\mathrm{A}}$ or by setting $\alpha$ to unity in (1).

When the imaged scene is immersed in a liquid and seen from the outside through a transparent wall, the projection is altered by refraction effects. In the simple case of a liquid-bathed scene observed through a plane wall, and under some restrictions relative to imaging configuration, however, the affine character of the projection can be preserved to a very good approximation, provided that the calibration step is performed in refraction conditions identical to those of the actual experiments (calibration target immersed in the fluid). This can be verified through a detailed analysis of ray refraction, and has been checked empirically to lead to negligible errors in De Backer (2001).

\section{3}

\section{Ray intersection and epipolar constraint}

Consider now a physical particle $\mathrm{P}$ having unknown position $\mathbf{r}_{\mathrm{P}}$ in $3 \mathrm{D}$ space. Let projections of the particle center on the left (focal point $A$ ) and right (focal point $B$ ) views have known image positions $R_{\mathrm{P}}^{(\mathrm{A})}\left(\mathrm{P}^{\prime}\right.$ on plane $\left.\Phi\right)$ and $\mathbf{R}_{\mathrm{P}}^{(\mathrm{B})}$ $\left(\mathrm{P}^{\prime \prime}\right.$ on plane $\left.\Psi\right)$. Referring to (5), the corresponding rays are given by parametric equations

$\mathbf{r}_{\mathrm{AP}}(\alpha)=\mathbf{r}_{\mathrm{A}}+\alpha \mathbf{s}_{\mathrm{AP}}$,

$\mathbf{r}_{\mathrm{BP}}(\beta)=\mathbf{r}_{\mathrm{B}}+\beta \mathbf{s}_{\mathrm{BP}}$,

where $\alpha$ and $\beta$ are the two free parameters. The 3D position of the particle can now be retrieved by finding the intersection of the two rays $\mathrm{AP}^{\prime}$ and $\mathrm{BP}^{\prime \prime}$ (Fig. 3). Due to imperfections of the imaging process, this intersection cannot be exact and instead we look for the point of closest encounter between the two rays. Let $\mathrm{M}$ and $\mathrm{N}$ be the points on both rays separated by the smallest interdistance. Their positions

$\mathbf{r}_{\mathrm{M}}=\mathbf{r}_{\mathrm{A}}+\alpha_{\mathrm{M}} \mathbf{s}_{\mathrm{AP}}$,

$\mathbf{r}_{\mathrm{N}}=\mathbf{r}_{\mathrm{B}}+\beta_{\mathrm{N}} \mathbf{s}_{\mathrm{BP}}$

are found by solving the following linear system in the two unknown parameters $\alpha_{\mathrm{M}}$ and $\beta_{\mathrm{N}}$ :
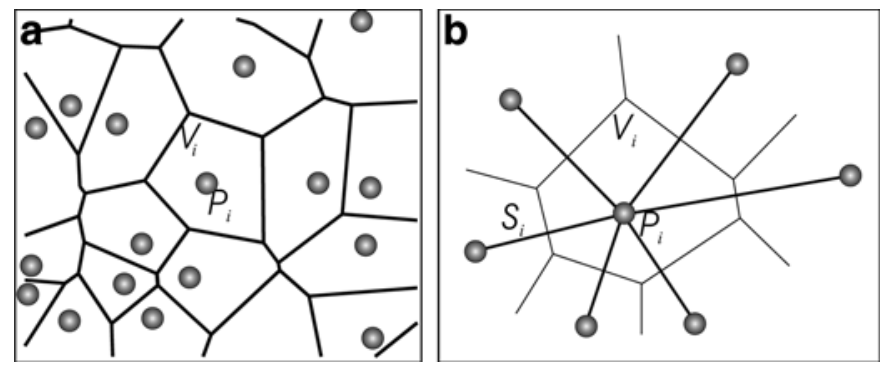

Fig. 4a-c. Voronoï diagrams (2D and 3D): a planar Voronoï diagram $\left\{\mathscr{V}_{i}\right\}$; b Voronoï vertex star $\mathscr{S}_{i}$ (thick lines) associated with cell $\mathscr{V}_{i}$ (thin lines) surrounding point $\mathscr{P}_{i}$; c a 3D Voronoï cell $v_{k}$ around point $p_{k}$;
$\left(\begin{array}{cc}\mathbf{s}_{\mathrm{AP}}^{\mathrm{T}} \mathbf{s}_{\mathrm{AP}} & -\mathbf{s}_{\mathrm{AA}}^{\mathrm{T}} \mathbf{s}_{\mathrm{BP}} \\ \mathbf{s}_{\mathrm{BP}}^{\mathrm{T}} \mathbf{s}_{\mathrm{AP}} & -\mathbf{s}_{\mathrm{BP}}^{\mathrm{T}} \mathbf{s}_{\mathrm{BP}}\end{array}\right)\left(\begin{array}{c}\alpha_{\mathrm{M}} \\ \beta_{\mathrm{N}}\end{array}\right)=\left(\begin{array}{l}{\left[\mathbf{r}_{\mathrm{B}}-\mathbf{r}_{\mathrm{A}}\right]^{\mathrm{T}} \mathbf{s}_{\mathrm{AP}}} \\ {\left[\mathbf{r}_{\mathrm{B}}-\mathbf{r}_{\mathrm{A}}\right]^{\mathrm{T}} \mathbf{s}_{\mathrm{BP}}}\end{array}\right)$.

The midpoint of the segment joining $\mathrm{M}$ and $\mathrm{N}$ constitutes an approximation of the true particle position $\mathrm{P}$. The length $\ell$ of the segment, on the other hand, measures the distance of closest encounter between the two rays and provides an estimate of the quality of the approximation. They are given by

$\mathbf{r}_{\mathrm{P}}=\frac{1}{2}\left[\mathbf{r}_{\mathrm{M}}+\mathbf{r}_{\mathrm{N}}\right]$

$\ell=\sqrt{\left[\mathbf{r}_{\mathrm{N}}-\mathbf{r}_{\mathrm{M}}\right]^{\mathrm{T}}\left[\mathbf{r}_{\mathrm{N}}-\mathbf{r}_{\mathrm{M}}\right]}$.

Suppose now that the only available information about a particle $\mathrm{P}$ is the position $\mathbf{R}_{\mathrm{P}}^{(\mathrm{A})}$ of its image $\mathrm{P}^{\prime}$ on the left view (plane $\Phi$ ). Can anything be said about projection $\mathbf{R}_{\mathrm{P}}^{(\mathrm{B})}$ of the same physical point on the right view (plane $\Psi$ )? Physical point $\mathrm{P}$ is known to belong to the plane $\mathrm{ABP}^{\prime}$ containing both focal points $\mathrm{A}$ and $\mathrm{B}$, and image $\mathrm{P}^{\prime}$ on the left view. Its parametric equation is

$\mathbf{r}(\alpha, \beta)=\mathbf{r}_{\mathrm{A}}+\alpha \mathbf{s}_{\mathrm{AP}}+\beta\left[\mathbf{r}_{\mathrm{B}}-\mathbf{r}_{\mathrm{A}}\right]$

where $\alpha$ and $\beta$ are again two free parameters. This plane $\mathrm{AP}^{\prime} \mathrm{B}$ is called the epipolar plane (see Fig. 3). Its intersection with the right image plane is a straight line $\epsilon \epsilon$, called the epipolar line, which constitutes the locus of all possible projections, in the right image plane, of a physical point having projection $\mathbf{R}_{\mathrm{P}}^{(\mathrm{A})}$ in the left image plane $\left(\mathrm{P}^{\prime}\right.$ on $\left.\Phi\right)$. Parametric equation $\mathbf{R}^{(\mathrm{B})}(\Gamma)$ of the epipolar line is easily found by projecting two points of the left ray $\mathrm{AP}^{\prime}$ on plane $\Psi$, via (1).

The true projection $\mathbf{R}_{\mathrm{P}}^{(\mathrm{B})}\left(\mathrm{P}^{\prime \prime}\right.$ on $\Psi$ ) is known to lie along this line, or, due to inevitable imperfections of the imaging process, to fall somewhere close to the epipolar line, i.e.,

$\mathbf{R}_{\mathrm{P}}^{(\mathrm{B})} \approx \mathbf{R}^{(\mathrm{B})}(\Gamma)$,

for a certain unknown value of parameter $\Gamma$. This restriction is known as the epipolar constraint, and can be used advantageously to guide the search for a correspondence between two stereoscopic views. It will be exploited below to accelerate the stereoscopic matching step.

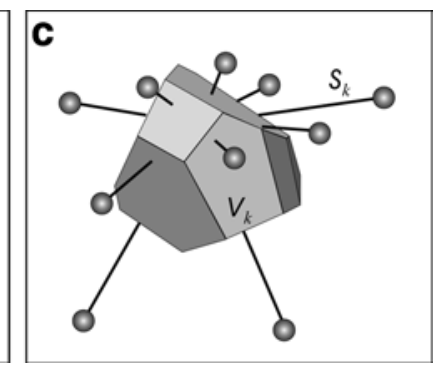

to each natural neighbor $(-)$ corresponds a particular facet of the Voronoï polyhedron $v_{k}$, perpendicular to a segment of the related vertex star $\mathscr{S}_{k}$ 


\section{3}

\section{Voronoï imaging methods}

With reference to Fig. $4 \mathrm{a}$, consider a set of feature points $\left\{\mathscr{P}_{i}\right\}$ placed at positions $\left\{\mathbf{R}_{i}\right\}$ in the $2 \mathrm{D}$ plane or $\left\{\mathbf{r}_{i}\right\}$ in $3 \mathrm{D}$ space. The Voronoï diagram is a geometrical construction that divides the space into a set of polytopes, or cells, surrounding each feature point. Each Voronoï cell $\mathscr{V}_{i}$ (in 2D) or $\mathscr{V}_{i}$ (in 3D) encompasses the region that lies closer to $\mathbf{R}_{i}$ (respectively $\mathbf{r}_{i}$ ) than to any other feature point of the set. The construction presents many useful properties, discussed in a general context in Okabe et al. (1992). In the present imaging context, three main properties are of interest:

1. The diagram organizes the dispersion of points into a network of neighborhood relations that can be used to speed up nearest-neighbor queries (Preparata and Shamos 1985). This will prove very useful to accelerate the stereoscopic matching step, which involves a repeated search for points of the image plane located in the vicinity of a given epipolar line.

2. The diagram defines a natural partition of space into non-overlapping regions, each containing a single particle. The inverse area or volume of the cell thus defines a local concentration estimate at the smallest possible scale. This can be used to sample statistical distributions in a finer and more robust way than by binning observations into an arbitrary partition (Bernardeau and van de Weygaert 1996). The property is exploited below to estimate particle concentrations.

3. The Voronoï diagram defines local patterns of neighboring feature points, which can be used as match templates (Ahuja 1982; Song et al. 1999). For moving dispersions of points, these patterns remain stable over a certain duration of time, a property exploited for 2D particle tracking by Capart et al. (2002). The present work extends this idea to 3D.

Stereoscopic matching, volumetric sampling, and particle-tracking operations are now outlined in three separate subsections.

\section{1 \\ Stereoscopic matching}

\subsection{1}

\section{Basic procedure}

For a single particle seen on two stereo views, the 3D particle position can easily be retrieved using the approximate ray intersection procedure of Sect. 2.3. To perform this operation when the imaged scene features a large number of particles, it is necessary first to solve the correspondence problem: find which particle image on one view corresponds to which one on the other. As required when all particles are identical, it can be solved on the basis of purely geometrical information by minimizing ray intersection discrepancies.

Consider two stereo images of a large number of identical particles (Fig. 1). From the particle-identification step, sets of 2D particle positions $\left\{\mathbf{R}_{i}^{(\mathrm{A})}\right\}$ and $\left\{\mathbf{R}_{j}^{(\mathrm{B})}\right\}$ have been located on the left and right views, respectively. Using the inverse projective transformation of Sect. 2.2, 3D rays can then be traced back through each of these image points. Ray bundles $\left\{\mathbf{r}_{i}^{(\mathrm{A})}(\alpha)\right\}$ and $\left\{\mathbf{r}_{J}^{(\mathrm{B})}(\beta)\right\}$ are thus obtained for the two views, and solving the correspondence problem amounts to finding a pairing $j(i)$ subject to the constraint that no more than one ray of one view can be paired with any one ray of the other (some rays can be left out, however, as a result of partial occlusion effects and differing viewing prisms). Once pairing $j(i)$ has been formed, the set of measured 3D positions $\left\{\hat{\mathbf{r}}_{i}\right\}$ is easily obtained from (13).

The pairing itself can be found by an optimization procedure, minimizing the objective function

$\sum_{i} \ell_{i j(i)}$

where the distance of closest encounter $\ell_{i j}$ given by (14) is taken as a "goodness-of-match" between any two rays $\mathbf{r}_{i}(\alpha)$ and $\mathbf{r}_{j}(\beta)$. This constitutes a standard bipartite graph optimization problem (e.g., Kim and Kak 1991), which is difficult (and computationally expensive) to solve thoroughly for large numbers of points. An approximate solution can however be found using the Vogel algorithm. It consists in considering for each ray the best match and the second best match, then constructing a reasonable global optimum by picking ray pairs in the order of maximum difference between first and second best choices.

\subsection{2}

\section{Voronoï epipolar screening}

For large numbers of particles, the procedure above becomes prohibitively expensive in terms of computational time and memory allocation. This is because it requires the computation of discrepancies $\ell_{i j}$ for all possible pairings of rays, and the handling of a full matrix in the Vogel optimization procedure. To limit those expenses, it is advantageous to conduct a first screening of possible match candidates by resorting to the epipolar constraint (16). This can be done very efficiently using the 2D Voronoï diagram.

With reference to Fig. 5a, consider a particle $i$ viewed on the left image at position $\mathbf{R}_{i}^{(\mathrm{A})}$. In the right image plane (Fig. 5b), let us construct the 2D Voronoï diagram $\left\{\mathscr{V}_{j}^{(\mathrm{B})}\right\}$ on the set of particle positions $\left\{\mathbf{R}_{j}^{(\mathrm{B})}\right\}$. The projection $\mathbf{R}_{j(i)}^{(\mathrm{B})}$ of particle $i$ in the right image is known to lie in the vicinity of epipolar line $\mathbf{R}_{i}^{(\mathrm{B})}(\Gamma)$. A simple way to screen match candidates is then to retain only those particle images $j$ that have their Voronoï cells $\mathscr{V}_{j}^{(\mathrm{B})}$ pierced by the epipolar line (see Fig. 5b, where 12 such candidates are marked). If particles are expected to lie within a limited depth range $\left[y_{\min } \ldots y_{\max }\right]$ (where $y$ is the normal to sidewall coordinate), parametric line $\mathbf{R}_{i}^{(\mathrm{B})}(\Gamma)$ becomes a line segment, and the set of match candidates can further be restricted (to the five candidates shown in black in the example of Fig. 5a, b).

If $N$ denotes the total number of visible particles, the procedure reduces considerably the number of candidates 


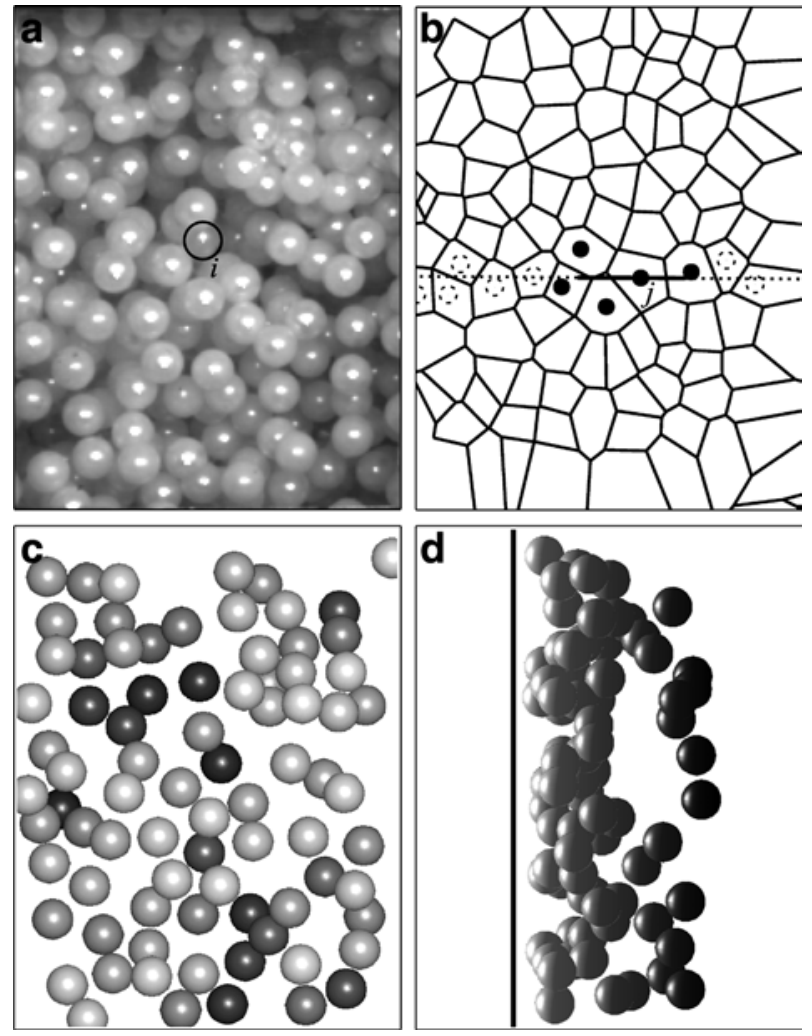

Fig. 5a-d. Principle of epipolar stereoscopic matching. a Considered particle center on the left view; $\mathbf{b}$ identification of the match candidates on image 2, whose Voronoï cells are pierced by the corresponding epipolar line (reduced to the segment in solid line); c reconstructed 3D positions of the particles. Color is assigned according to depth, bright for near-wall particles, darker for deeper ones; $\mathbf{d}$ side view of $\mathbf{c}$, solid line indicating wall position

to be considered from $N$ to $\sim \sqrt{N}$ (screening along a line instead of the full area). The 2D Voronoï diagram must be computed only once at a cost of $\sim M \log N$ operations (Okabe et al. 1992). Epipolar line piercing then requires only a nearest-neighbor search and neighbor-byneighbor propagation within the Voronoï diagram, for a combined cost of $\sim \sqrt{N}$. Overall, the Voronoï epipolar screening reduces the cost of stereo matching from $\sim N^{2}$ to $\sim N^{3 / 2}$. For the experiments described in Sect. 4 , this amounts to a 10-30-times speed-up over the brute-force approach.

Figure $5 \mathrm{c}$ and $\mathrm{d}$ show the reconstructed $3 \mathrm{D}$ dispersion of particles resulting from application of the complete stereo matching procedure. Starting from sets of image positions $\left\{\mathbf{R}_{i}^{(\mathrm{A})}\right\}$ and $\left\{\mathbf{R}_{j}^{(\mathrm{B})}\right\}$, the procedure is seen to be successful at pinpointing the $3 \mathrm{D}$ positions $\left\{\hat{\mathbf{r}}_{i}\right\}$ of most of the visible particles. An indication of the robustness of the approach is that partial occlusion relationships (visible by eye on the image but not exploited in the analysis) are well conserved by the reconstruction. It is also seen in Fig. 5d that, for such a densely packed dispersion, occlusion effects prevent the retrieval of particle positions beyond a depth of a few grain diameters. This issue is addressed again in the next subsection.

\section{2 \\ Volumetric sampling}

\subsection{1 \\ Binning and Voronoï sampling estimates of volumetric concentration}

Supposing one can pinpoint a full set of 3D particle positions $\left\{\mathbf{r}_{k}\right\}$ inside the viewing region, this set can be exploited to yield further spatial information about the granular dispersion. The quantity of most interest is the local volumetric concentration $\phi(\mathbf{r})$. The simplest way of obtaining estimates for $\phi$ is to subdivide the viewing volume into an arbitrary spatial partition $\left\{\mathscr{W}_{j}\right\}$, then to count the number of particle centroids $n_{j}$ falling into each box $\mathscr{W}_{j}$ of the partition. The local concentration in each box is then estimated as:

$\hat{\phi}_{j}=\frac{n_{j} V_{\mathrm{p}}}{\operatorname{volume}\left(\mathscr{W}_{j}\right)}$

where $V_{\mathrm{P}}=\frac{1}{6} \pi d^{3}$ is the volume of a single solid particle. This is the so-called "binning" procedure, widely used to estimate statistical distributions.

Instead of defining an arbitrary partition, an alternative approach makes use of the 3D Voronoï diagram. By construction, the Voronoï diagram partitions the region into a set of cells $\left\{\mathscr{V}_{k}\right\}$, each one containing a single particle. Local concentrations can then be estimated as:

$\hat{\phi}_{k}=\frac{V_{\mathrm{P}}}{\operatorname{volume}\left(\mathscr{V}_{k}\right)}$.

Advantages of the approach are that Voronoï partition $\left\{\mathscr{V}_{k}\right\}$ is more natural than arbitrary partition $\left\{\mathscr{W}_{j}\right\}$, and that concentrations are sampled at the locations $\left\{\mathbf{r}_{k}\right\}$ where particle velocities are defined. To apply such estimates in the case of a dispersion bounded by a plane side-wall, it is convenient to construct an extended Voronoï diagram on the basis of the set $\left\{\mathbf{r}_{k}\right\}$ complemented by set $\left\{\mathbf{r}^{\prime}{ }_{k}\right\}$ of its mirror images on the other side of the wall (refer to Fig. 8a, discussed more in details in Sect. 3.2.3). This guarantees that near-wall cells will be bounded by facets belonging to the plane boundary, hence allowing concentration estimate (19) to take the wall into account.

\subsection{2}

\section{Occlusion effects}

Unfortunately, for a dense dispersion of opaque grains, the set of measured particle positions $\left\{\hat{\mathbf{r}}_{i}\right\}$ picked up by the stereo procedure is not the full physical set $\left\{\mathbf{r}_{k}\right\}$, but a subset of this including only the particles which are visible under both camera viewpoints. Occlusion effects intervene, whereby particles in the front hide from view particles in the back. To get a feel for how occlusion affects concentration estimates, it is useful to analyze first a simple model, introduced by Capart et al. (2002) in a monocular context and extended here to stereo acquisition.

Let us assume a 3D dispersion of spherical grains of diameter $d$, characterized by constant volumetric concentration $\phi$ in the near-wall region. Disregarding excluded volume effects due to impenetrability of the solid particles and side-wall, one may assume that the 

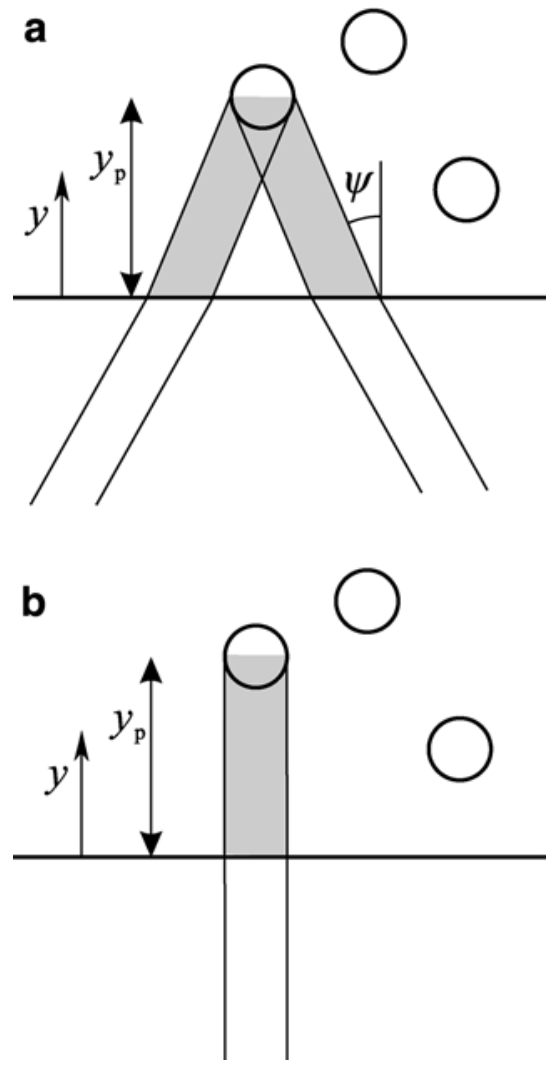

Fig. 6a, b. Occlusion analysis. Shaded volumes are the regions that must be free of occluding grains for a given particle center to be seen under: a two stereo views; $\mathbf{b}$ a single monocular view

particle centroids are distributed inside the 3D volume according to a homogeneous Poisson process, with an average number of particles per unit volume equal to $\mu_{\mathrm{P}}=\phi V_{\mathrm{P}}^{-1}$. Considering the idealized stereo imaging configuration shown in Fig. 6a, a particle centroid will be visible on both stereo views only if no other particle center invades the two viewing cylinders shown as hatched surfaces. Given the depth of the particle $y_{\mathrm{p}}$ and the viewing angle $\psi$ under which it is seen by each camera, the hatched volume can be approximated by

$$
\begin{aligned}
& V\left(y_{\mathrm{P}}\right) \approx \frac{\pi d^{2}}{4}\left(y_{\mathrm{P}}+y_{\mathrm{P}}^{2} \frac{\tan \psi}{d}\right) \quad \text { if } y_{\mathrm{P}} \leq \frac{d}{2 \tan \psi}, \\
& V\left(y_{\mathrm{P}}\right) \approx \frac{3 \pi d^{3}}{16 \tan \psi}+\frac{\pi d^{2}}{2}\left(y_{\mathrm{P}}-\frac{d}{2 \tan \psi}\right) \quad \text { if } y_{\mathrm{P}} \geq \frac{d}{2 \tan \psi},
\end{aligned}
$$

in which angle $\psi$ is assumed to be small, and where the complex sub-volume of intersection of the two cylinders is only roughly estimated. The probability that a particle is viewed under both viewpoints is then given by Poisson distribution (e.g., Adrian 1991; Okabe et al. 1992)

$\mathrm{P}[0$ particle in $V]=\frac{\left(\mu_{\mathrm{P}} V\right)^{0}}{0 !} \exp \left(-\mu_{\mathrm{P}} V\right)=\exp \left(-\mu_{\mathrm{P}} V\right)$.

The average volumetric concentration of visible particles $\hat{\phi}$ is thus only a fraction (22) of the actual one $\phi$ and can be expressed as the following function of depth $y$ :

$\hat{\phi}(y)=\phi \mathrm{P}[0$ particle in $V(y)]=\phi \exp \left(-\phi \frac{V(y)}{V_{\mathrm{P}}}\right)$.

As expected, the two concentrations are equivalent right next to the side-wall $(y \approx 0)$. Away from the side-wall, the concentration of visible particle drops as a result of the rising probability of occlusion.

Figure 7 compares the predictions of (23) with measurements $\hat{\phi}_{j}\left(y_{j}\right)$ obtained from actual fluidization cell experiments. The measured functions were obtained by binning actual stereo measurements into partition $\left\{\mathscr{W}_{j}\right\}$ given by

$\mathscr{W}_{j} \equiv\left\{\mathbf{r} \mid y_{j}-\frac{1}{2} \delta y<y<y_{j}+\frac{1}{2} \delta y\right\}$,

i.e., layers of small thickness $\delta y$ parallel to the side-wall, then applying (18). The measured profiles of Fig. 7b exhibit clear humps and troughs. This is not an artefact, but a consequence of excluded volume effects (neglected in the simple occlusion analysis). Physical particle centers are
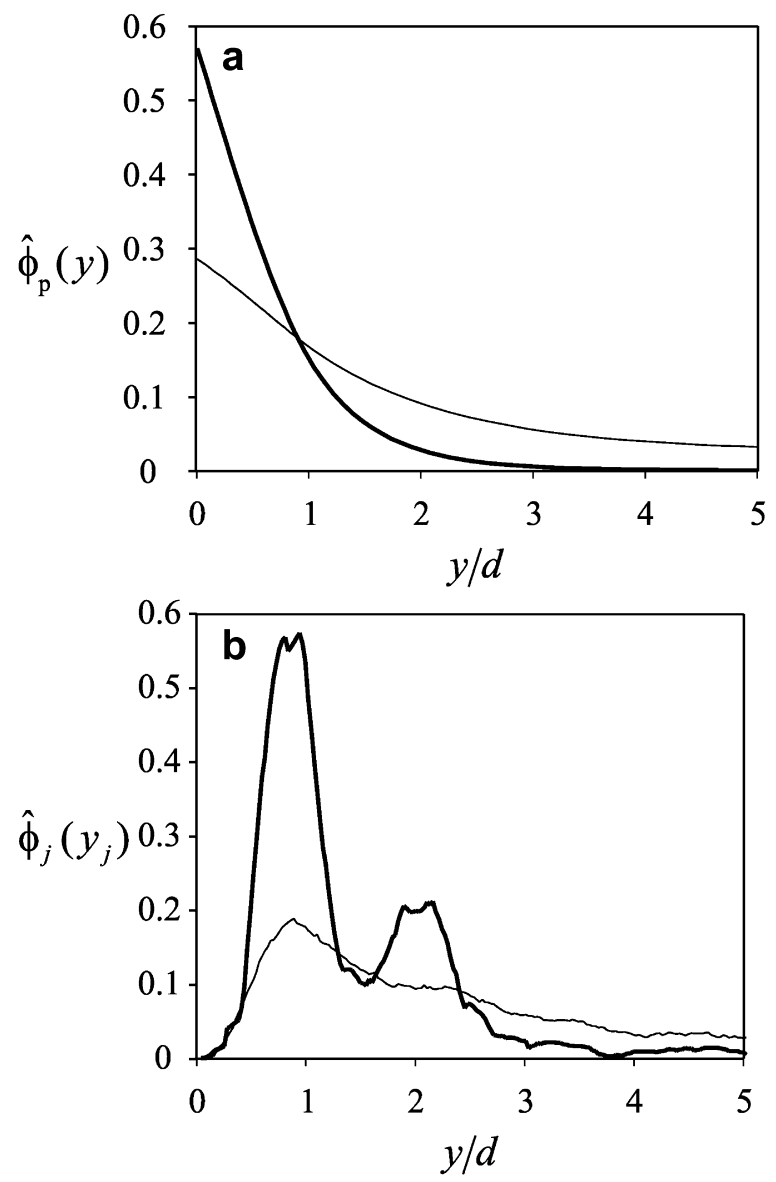

Fig. 7a, b. Distribution of visible particles centers in function of the depth (normal to wall) for two characteristic concentrations (thick line $57 \%$, thin line $26 \%$ ). a Theoretical distribution derived from a 3D Poisson process; $\mathbf{b}$ experimental distribution gathered from fluidization cell tests. Wall position corresponds to the left of the graphs; intervals between successive graduations on the horizontal axis correspond to one particle diameter 
located preferentially at depths corresponding to certain multiples of particle diameter $d$, in a crystalline-like order induced by the side-wall boundary. These effects are especially strong for the denser packing $(\phi \approx 0.57)$. The decay curves, which underlie these quasi-periodic variations, on the other hand, show the effect of occlusion. This exponential-like decay is seen in Fig. 7 to be reasonably well captured by the simple occlusion model introduced above. In particular, it is observed on both sets of results that away from the side-wall, the concentration of visible particles may actually be higher for the case of lower concentration $(\phi \approx 0.26)$ than for the densely packed case $(\phi \approx 0.57)$. This is entirely a result of occlusion, and explains why one must be careful in trying to derive actual concentrations $\phi$ from measured concentrations of visible grains $\hat{\phi}$.

Another signal that caution must be exercised comes from examining the consequences of the simple Poisson occlusion model outlined above in the case of monocular imaging (Fig. $6 \mathrm{~b}$ ). In that case, the viewing region, which must be free of occluding particles, simplifies to a cylinder, and the surface particle density $\eta$ obtained by projecting the images of all the visible particles onto the side-wall (i.e., as seen by the monocular camera) is given by (Capart et al. 2002)

$\hat{\eta}=\frac{4}{\pi d^{2}}$.

The key feature of (25) is that the resulting estimate $\hat{\eta}$ is independent of the actual particle concentration $\phi$. The result is surprising because it is very tempting to assume that one can estimate grain concentrations by simply counting the surface density of particles seen on monocular images, then resorting to some ad hoc calibration to convert $\hat{\eta}$ into $\phi$. This obvious estimate, however, is geometrically completely insensitive to $\phi$. Only because of attenuation in illumination with depth does some sensitivity appear, making the measurements hinge upon illumination conditions, which are usually not well controlled in the laboratory. Such questionable estimates are nevertheless found in the literature including, we must confess, some of the authors' earlier work (Capart et al. 1997).

\subsection{3}

\section{Surface Voronoï sampling of the near-wall concentration}

Because of occlusion, one should not expect to be able to measure concentrations inside the bulk of the opaque disperse phase. What should be possible, however, on the basis of the stereo position results $\left\{\hat{\mathbf{r}}_{i}\right\}$, is to estimate the near-wall volumetric concentration

$\hat{\phi}_{0} \cong \phi(y \approx 0)$.

As seen in Fig. 7, occlusion effects are so severe for large concentrations that some care must be exercised even to attain this more modest objective. One possibility examined in a preliminary work (Spinewine et al. 2000) is to resort to estimate

$\hat{\phi}_{i}=\frac{V_{\mathrm{P}}}{\operatorname{volume}\left(1 V_{i}\right)}$
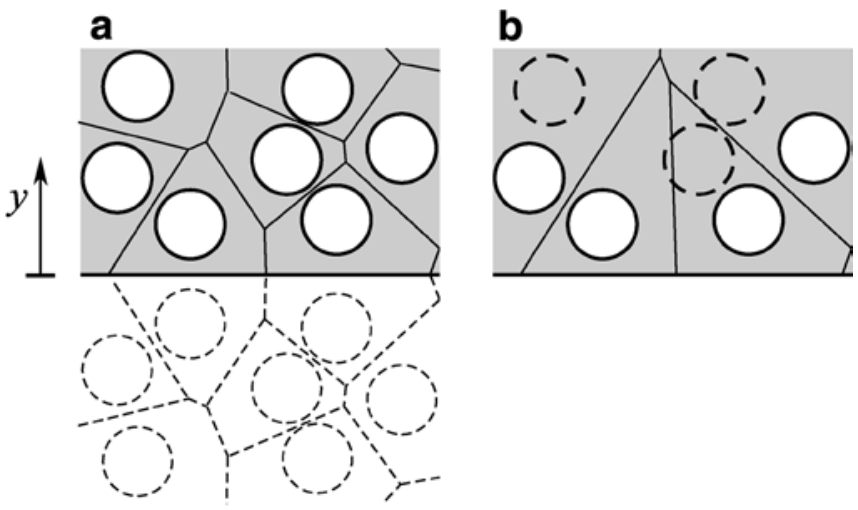

Fig. 8a, b. Volumetric sampling: a mirror image construction of a Voronoï diagram in which the plane side-wall is embedded;

b incomplete near-wall Voronoï cell closures resulting from occlusion of back row particles. (Note: whereas 3D diagrams are actually used in the experiments, 2D analogues are shown here for illustration purposes)

where the $\left\{\mathscr{V}_{i}\right\}$ are the 3D Voronoï cells constructed on the basis of the $\left\{\hat{\mathbf{r}}_{i}\right\}$ and their mirror images $\left\{\hat{\mathbf{r}}_{i}\right\}$ (Fig. 8a). To limit the effects of occlusion, estimates (27) are then sampled only within the near-wall Voronoï cells, i.e., the cells $\left\{\mathscr{V}_{k}\right\}$, which share a face with the side-wall. This yields reasonable results up to moderate concentrations, for which particles are visible till a depth of several diameters. However, for larger concentrations (beyond $\phi \approx 0.4$ ), some second- and third-row particles become unseen (Fig. 8b), leading to unrealistically large volume of front-row Voronoï cells. As a consequence, the concentration for dense packing is significantly under-predicted by (27).

To remedy this problem, it is proposed to resort to the following modified surface particle-density estimate:

$\hat{\eta}_{0, i}=\frac{1}{\operatorname{area}\left(\mathscr{A}_{0}\left(\mathscr{V}_{i}\right)\right)}$

where $\mathscr{A}_{0}\left(\mathscr{V}_{i}\right)$ is the plane face $\mathscr{A}_{0}$ which the $3 \mathrm{D}$ cell $\mathscr{V}_{i}$ shares with the wall boundary (or equivalently shares with its mirror image). The advantage of measuring a surface density rather than volumetric density is that the frontal face of the near-wall Voronoï cells is not affected by occlusion. Estimate (28) is further assumed to hold uniformly over face $\mathscr{A}_{0}\left(\mathscr{V}_{i}\right)$. When averaging over a certain extent of the wall surface, with area $A$ large with respect to the area of an individual cell face, we have that

$\left\langle\hat{\eta}_{0}\right\rangle \approx \frac{n_{0}}{A}$

where the brackets \langle\rangle denote a spatial average, and $n_{0}$ is the number of near-wall particles picked up within area $A$, i.e., the number of visible particles which have a Voronoï cell sharing a face with the wall within that area.

Stereoscopic surface estimate (29) differs from monocular surface estimate (25) in one fundamental regard. In the monocular case (25), all visible particles are counted regardless of the depth. In the stereoscopic case (29), by contrast, only the near-wall particles (defined in the Voronoï sense) are counted. The stereo near-wall estimate is therefore biased neither by occlusion effects (which 
affect the volumes of the 3D Voronoï cells) nor by visible particles located away from the wall (which affect the monocular estimate).

\subsection{4}

\section{Monte Carlo estimation of the stereological coefficient}

A final step is required, which is to convert surface estimate $\left\langle\hat{\eta}_{0}\right\rangle$ into volumetric estimate $\hat{\phi}_{0}$. This relationship can be predicted a priori if the $3 \mathrm{D}$ distribution of particle centers within the measurement volume is assumed to derive from a homogenous Poisson process, defining once again $\mu_{\mathrm{p}}$ as the average number of particles per unit volume. In that case, it follows from dimensional considerations that the relationship between $\left\langle\eta_{0}\right\rangle$ and $\mu_{\mathrm{p}}$ must be of the form

$\left\langle\eta_{0}\right\rangle=\chi \mu_{\mathrm{p}}^{2 / 3}$

where $\left\langle\eta_{0}\right\rangle$ is the expected surface density of near-wall particles and $\chi$ is a non-dimensional constant: the so-called stereological coefficient.

While it is not impossible that an exact value for constant $\chi$ could be derived theoretically, this is beyond our level of expertise. We thus resorted to Monte Carlo simulations (e.g., Ross 1990) to obtain an approximate value. Repeated simulation runs were conducted, each run involving the following steps:

1. With the aid of a random number generator, a 3D Poisson process of given intensity $\mu_{\mathrm{p}}$ is simulated within a box of finite size;

2. The resulting dispersion of points is reflected on the other side of one of the box walls (= the side-wall);

3. The 3D Voronoï diagram is computed and the near-wall cells identified;

4 The surface density $\left.\eta_{0}\right\rangle$ is estimated by counting nearwall particles or averaging the areas of the cell faces $\mathscr{A}_{0}\left(\mathscr{V}_{i}\right)$ coinciding with the mirror plane.

For step 4, estimates are sampled in a central region only in order to avoid boundary effects. Except for step 1, the procedure closely emulates the one applied to the actual stereo measurements. A batch of 1,000 Monte Carlo runs was carried out, each run involving $\sim 2,000$ particles. The result of these computations is $\chi=0.92 \pm 0.01$, where the bounds correspond to a $95 \%$ confidence interval (see Ross 1990).

Recalling that $\mu_{\mathrm{p}}=\phi V_{\mathrm{p}}^{-1}$, relation (30) implies

$\hat{\phi}_{0}=V_{\mathrm{P}}\left(\frac{\left\langle\hat{\eta}_{0}\right\rangle}{\chi}\right)^{3 / 2}$,

which is our estimate for the near-wall volumetric concentration $\hat{\phi}_{0}$ in terms of the averaged wall-sampled surface number density $\left\langle\hat{\eta}_{0}\right\rangle$. Strictly speaking, relation (31) with constant $\chi \approx 0.92$ should be expected to hold only in the dilute limit. For dense dispersions, excluded volume effects will make the distribution of particle centers differ from the results of a Poisson process. As verified by the experiments of Sect. 4.1, it will turn out nevertheless to constitute a reasonable approximation over the entire range of concentrations. Such a favorable situation is encountered in other stereology problems (Lorz 1990; Okabe et al. 1992). Estimate (31) is remarkable in that it derives from purely geometrical considerations. Up to moderate concentrations, it requires no ad hoc calibration. This contrasts with the much less favorable situation faced when trying to extract concentration estimates from monocular images (Capart et al. 2002).

\section{3}

\section{Pattern-based particle tracking}

The third operation that exploits the properties of the Voronoï diagram is the particle-tracking step. Sets of 3D particle positions at successive times are first acquired by repeated application of stereo matching to each frame of a movie sequence. Let $\left\{\mathbf{r}_{i, m}\right\}$ and $\left\{\mathbf{r}_{i, m+1}\right\}$ be two such sets of particle positions sampled at successive times $t_{m}$ and $t_{m+1}=t_{m}+\Delta t$. Particle velocities $\mathbf{v}_{i}\left(t_{m+1 / 2}\right)$ can be estimated by expression

$\mathbf{v}_{i, m+1 / 2}=\frac{\mathbf{r}_{j(i), m+1}-\mathbf{r}_{i, m}}{\Delta t}$,

provided one can first "connect the dots", and establish a pairing $j(i)$ between positions $\mathbf{r}_{i, m}$ and $\mathbf{r}_{j(i), m+1}$ belonging to one and the same physical particle. When dealing with a moving dispersion of many identical particles, the main problem consists in establishing this correspondence: finding which particle on one snapshot corresponds to which one on the next. The particle-tracking problem can thus be seen as a time-domain variant of the stereo matching problem addressed previously.

For dilute particle dispersions or slow motion, the correspondence problem can easily be solved simply by pairing together the particles on one frame and the next, which are nearest to each other (see, e.g., Guler et al. 1999). For dense dispersions or rapid motion, however, legitimate pairing candidates may travel further away and the minimum displacement criterion breaks down. An alternative approach derives from the following observation: while individual particles are identical to each other, the local arrangements that they form with their neighbors are unique and may be preserved by the flow long enough to serve as a basis for tracking. Particle pairing can then be performed based on pattern similarity.

Following Capart et al. (2002), we again resort to the Voronoï diagram to implement such pattern-based tracking. Nearby particles are paired according to the geometrical similarity of their Voronoï cells. This similarity is estimated as follows: "stars" are first constructed by connecting a given particle center to its Voronoï neighbors (i.e., the particles with which it shares a cell face), as illustrated in Fig. $4 \mathrm{~b}$ and $\mathrm{c}$ for both the $2 \mathrm{D}$ and $3 \mathrm{D}$ cases. The stars belonging to two pairing candidates can then be compared for goodness-of-fit by making their centers coincide and measuring the distances between the star extremities. Once these "goodness-of-fit" indicators are available for all possible pair candidates, a global optimum problem can be defined and solved in the same fashion as in the stereo matching case (see Sect. 3.1 and Eq. 17).

For two dimensions, the overall method is illustrated in Fig. 9 for a plane granular flow. While graphical representation is harder in $3 \mathrm{D}$, the algorithms themselves generalize straightforwardly to the third dimension. The 

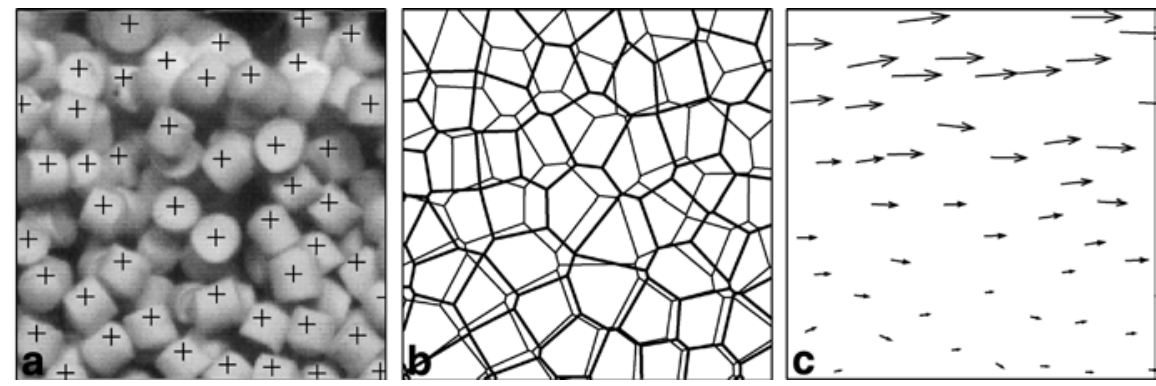

Fig. 9a-c. Pattern-based velocimetric tracking: a original image fragment and particle positions; $\mathbf{b}$ Voronoï diagrams built on sets of particle positions at two successive time instants; c velocity vectors built by matching of the local Voronoï patterns. (Note: whereas 3D diagrams are actually used in the experiments, 2D analogues are shown here for illustration purposes)

reader is referred to Capart et al. (2002) for a detailed presentation and comparison with alternative approaches. Three-dimensional applications and results are detailed further below.

\section{4}

\section{Liquid-granular flow applications}

The Voronoï imaging methods presented in the last two sections are now applied to two different cases of immersed particulate flows, each aimed at highlighting particular aspects of the techniques. Fluidization cell experiments are chosen as a first test case, ideally suited for validation of concentration estimates. The steady uniform flow of a water-granular mixture down an inclined open channel was selected as a second test case. Featuring strong gradients in both solid fraction and granular velocities, this second case constitutes a challenging application for both velocity and concentration measurements.

\section{1}

\section{Fluidization cell experiments}

\subsection{1}

\section{Principle and set-up}

The proposed imaging techniques are now applied to fluidization cell tests. The principle of the tests is as follows. Subject to an ascending water current, a layer of loosely packed grains expands into a fluidized suspension. The concentration adapts to the water flux until the mean drag balances the submerged weight of the grains. In this fluidized state, the suspended particles undergo weakly correlated fluctuating motions, exploring a variety of spatial arrangements. Most important in the present context, the average concentration of particles is spatially uniform throughout the fluidized layer. Different concentrations can be obtained simply by tuning the fluid flow. Beyond their intrinsic interest, such homogeneous states of known concentration are ideal for testing concentration measurement methods.

The device used for the experiments is presented in Fig. 10. The cylindrical fluidization cell has a height of $25 \mathrm{~cm}$ and an inner diameter of $10 \mathrm{~cm}$. A $5 \mathrm{~cm}$ deep layer of small lead spheres is placed at the bottom of the cell to diffuse the incoming water current and provide uniform fluid velocity throughout the cross-section. Above this heavy static layer comes the granular bed to be fluidized. It is composed of light spheres (artificial pearls) of relative density $\rho_{s} / \rho_{w}=1.048$ and diameter $D=6.1 \mathrm{~mm}$. To allow visualization without optical distortion, a plane rectangu- lar observation window of dimensions $5 \times 10 \mathrm{~cm}$ is fitted to the cell wall.

The evolving granular dispersion is imaged by two synchronized CCD cameras placed in a stereoscopic arrangement. The sensors, each $256 \times 256$ pixels in size, are positioned approximately $35 \mathrm{~cm}$ away from the cell, and mounted $\pm 25 \mathrm{~cm}$ apart from each other. The angle between the two viewpoints is thus around $20^{\circ}$. Both lenses have a focal distance of $16 \mathrm{~mm}$. Particle diameters span around 10 pixels. The resulting positioning error on particle centroids is estimated to be of the order of $0.2 \mathrm{~mm}$.

The viewpoint calibration procedure sketched in Sect. 2.2 is carried out by imaging an "open-book"shaped dihedron placed within the cell filled with water. A total of 56 calibrations points is used for the least-square derivation of matrixes $A^{(A)}, A^{(B)}$ and vectors $\mathbf{b}^{(A)}, \mathbf{b}^{(\mathrm{B})}$ needed to determine the left $(A)$ and right $(B)$ viewpoints. The world coordinate system $(x, y, z)$ is as follows: the $x-z$ plane coincides with the cell wall ( $x$ taken horizontally), and $y$ represents the out-of-plane horizontal coordinate (i.e., depth inside the cell).

Two independent series of tests were carried out under slightly different camera and lighting conditions. For each series, measurements were performed for a number of experimental runs corresponding to different solid concentrations (see Table 1). The Richardson-Zaki empirical correlation (Richardson and Zaki 1954) was found to describe well the relation between fluidization velocity and concentration.

In order to derive granular velocities and concentrations, the sets of simultaneous images are first preprocessed to
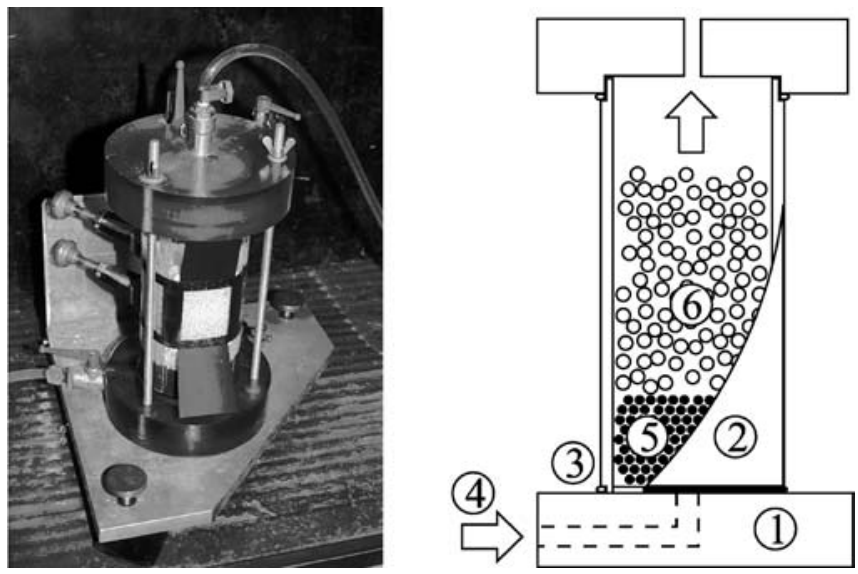

Fig. 10. The fluidization cell: 1 bounding plates; 2 Acrylic glass cell shaft; 3 impermeable joints and fixation screws; 4 incoming water flow; 5 layer of lead spheres to diffuse water current; 6 fluidized bed 
Table 1. Test conditions for the fluidization experiments

\begin{tabular}{|c|c|c|c|c|c|c|c|c|c|c|c|}
\hline \multicolumn{12}{|l|}{ First series of tests } \\
\hline Run number & 1 & 2 & 3 & 4 & 5 & 6 & 7 & 8 & 9 & 10 & 11 \\
\hline Fluidization velocity $(\mathrm{cm} / \mathrm{s})$ & 1.97 & 1.05 & 2.46 & 1.78 & 2.20 & 2.54 & 0.93 & 0.72 & 1.18 & 1.84 & 2.95 \\
\hline Bulk concentration $(\%)$ & 37.9 & 52.1 & 30.7 & 40.5 & 33.6 & 29.2 & 55.7 & 59.0 & 43.5 & 38.3 & 26.6 \\
\hline \multicolumn{12}{|l|}{ Second series of tests } \\
\hline Run number & 12 & 13 & 14 & 15 & 16 & 17 & 18 & 19 & 20 & 21 & 22 \\
\hline Fluidization velocity $(\mathrm{cm} / \mathrm{s})$ & 0.90 & 1.11 & 1.26 & 1.70 & 1.96 & 2.36 & 2.57 & 1.08 & 1.87 & 0.95 & 1.48 \\
\hline Bulk concentration $(\%)$ & 55.7 & 50.7 & 47.2 & 41.2 & 37.1 & 32.4 & 29.3 & 51.4 & 38.4 & 54.3 & 43.6 \\
\hline
\end{tabular}

pinpoint particle positions in the two image planes. Stereoscopic ray matching and intersection is then used to retrieve the $3 \mathrm{D}$ positions of the grains. These procedures were described and illustrated in Sect. 2. We now focus on the concentration estimation process.

\subsection{2}

\section{Concentration estimation}

For every fluidization velocity, the height of the fluidized layer furnishes a direct and fairly accurate measure of the bulk concentration, which can serve as validation of the imaging estimates. The latter are extracted from uncorrelated snapshots acquired at a very low frame rate. Since the number of particles seen on each image is limited, this is needed to sample a variety of particle configurations and obtain good statistics by averaging concentration measurements over a number of images ( 16 and 30 images per run were used, respectively for the first and second series of tests).

Figure 11a presents a plot of the bulk concentration versus its imaging estimate. The latter is obtained by positioning the particle centers, constructing their 3D Voronoï diagram, and sampling the surface density of near-wall particles using estimate (29). Coefficient $\chi=0.92$ derived from the Monte Carlo simulations is then used in (31) to convert surface number density $\left\langle\hat{\eta}_{0}\right\rangle$ into volumetric concentration $\hat{\phi}_{0}$. In Fig. 11a, the imaging results are seen to be close to the line of perfect agreement up to moderate concentrations $(\phi \approx 0.4)$. For higher concentrations, the theoretically based imaging estimate $\hat{\phi}_{0}$ begins to underestimate the actual concentration $\phi$. This departure from perfect agreement most likely arises due to excluded volume effects, which induce a quasi-crystalline arrangement of the grains at dense packing. The Poisson process assumption adopted to derive relation (31) and compute the value of stereological coefficient $\chi$ then breaks down.

Remarkably, however, the discrepancies are not very severe. Without any adjustment, the predicted relation remains accurate to $10 \%$ over the entire range. Furthermore, the data sets obtained from the two independent series of tests are very consistent with each other. Based on the fluidization data, an empirically adjusted estimate can be proposed in the form

$\hat{\phi}_{0}^{*}=\frac{1}{\kappa} \tanh ^{-1}\left(\kappa \hat{\phi}_{0}\right)$

where $\kappa$ is a dimensionless coefficient and value $\kappa=1.2$ provides a good fit to the measurements. The functional form of (33), involving an inverse tangent, reduces to (31) in the dilute limit, and introduces a significant correction only at high concentrations $(\phi>0.4)$ for which the Poisson process assumption breaks down. The data are plotted again in Fig. 11b based on this empirically adjusted relation. The apparent robustness and sensitivity of the stereoscopic imaging estimate contrasts with the much less favorable properties of the monocular imaging indicators examined in Capart et al. (2002).

\subsection{3}

\section{Three-dimensional particle motions}

The evolution of the particle arrangement in time can further be characterized by following the 3D motions of the imaged particles using the procedures of Sect. 3.3. In this application, the movement is relatively slow and the tracking procedure easy to carry out. A more challenging application for the tracking component of the algorithms is
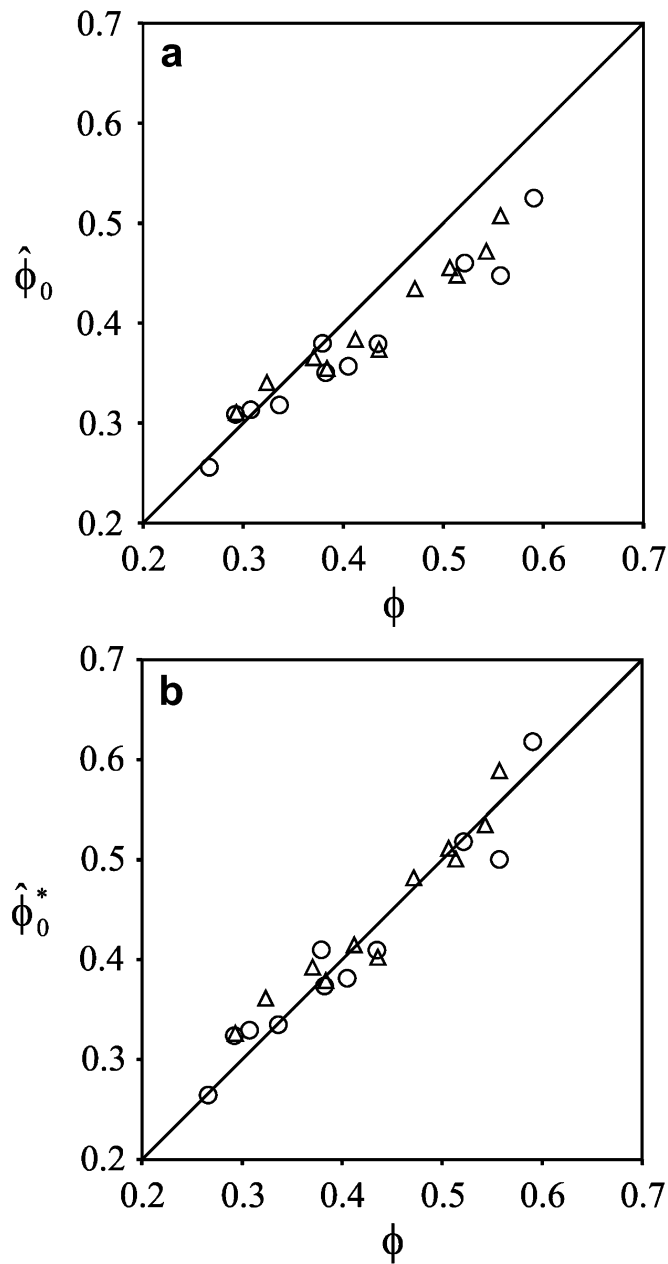

Fig. 11a, b. Granular concentration: bulk measurements vs. imaging estimate. $\bigcirc$ first series of tests; $\triangle$ second series of tests; - line of perfect agreement. a imaging estimate $\hat{\phi}_{0}(31) ; \mathbf{b}$ empirically adjusted estimate $\hat{\phi}_{0}^{*}$ (33) with $\kappa=1.2$ 
the rapidly sheared example presented in the next section. The key component here is the stereoscopic positioning step. Reliable 3D positions must be measured in order to record continuous trajectories. To damp out high-frequency noise due to the finite sensor resolution, particle displacements are averaged over nine successive frames (with a frame rate of $200 \mathrm{fps}$, this corresponds to an interval of $0.04 \mathrm{~s}$, which was checked to be much less than the average time between collisions) to yield velocity and trajectory measurements. Sample particle paths obtained for a fluidization concentration $\phi=0.38$ are presented in Fig. 12 . The fluctuating motions of neighboring grains are influenced by each other on a scale of a few particle diameters. This appears typical of hydrodynamic effects whereby conjugate motions of the embedding fluid transmit the influence of particle motions over a certain distance.

Mean squared velocity fluctuations along the three spatial directions $x, y, z$ are plotted in Fig. 13 for different fluidization concentrations $\phi$. The observed velocity fluctuations are greater at lower concentrations, for which particles have more freedom to move around. Lower concentrations also correspond to a faster fluidization flux and greater transfer of kinetic energy to the fluctuating motions. Vertical fluctuations $\left\langle w^{\prime 2}\right\rangle$ are found to be slightly larger than the horizontal fluctuations $\left\langle u^{\prime 2}\right\rangle$ and $\left\langle v^{\prime 2}\right\rangle$. Deviations from isotropy are not very large, however. A significant observation is that, despite the presence of the side-wall, the in-plane and out-of-plane horizontal fluctuations have similar magnitudes.

\section{2}

\section{Uniform debris-flow experiments}

\subsection{1}

\section{Principle and set-up}

The second test case is an open-channel flow of a highly concentrated liquid-granular mixture imaged through the
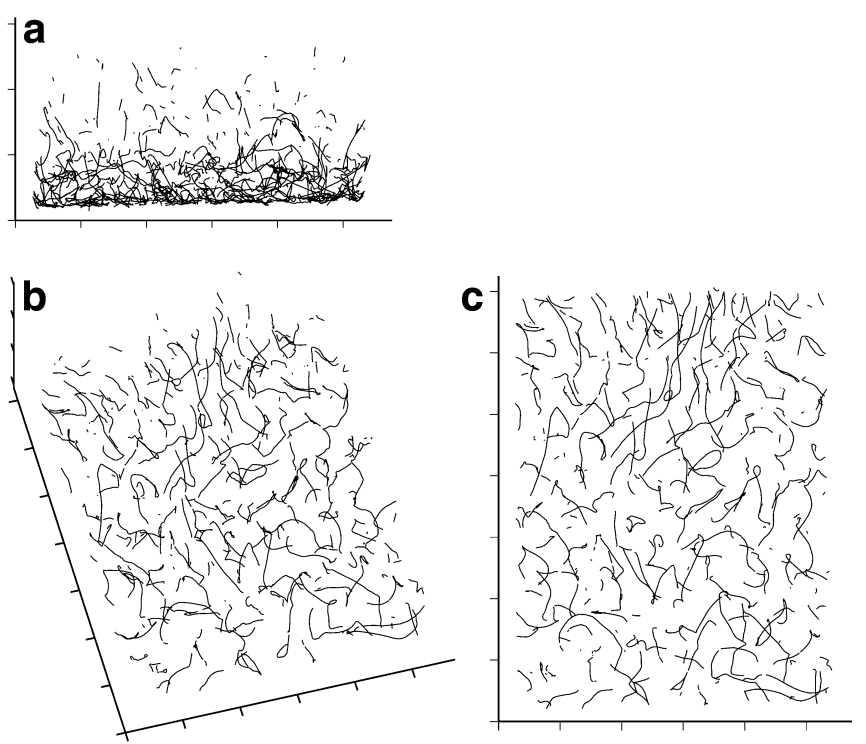

Fig. 12a-c. Example of granular trajectories obtained for a fluidization cell experiment at a bulk concentration of $38 \%$ (run \#1 of the first series). a Top view, in the $x-y$ plane; $\mathbf{b} 3 \mathrm{D}$ view; $\mathbf{c}$ front view, in the $x-z$ plane

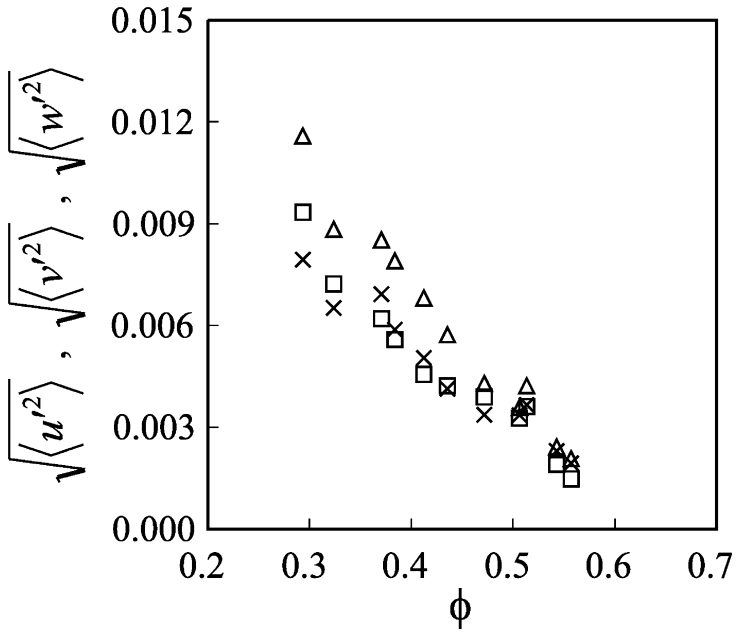

Fig. 13. Velocity fluctuations vs. bulk concentration. $\square$ horizontal component $\sqrt{\left\langle u^{\prime 2}\right\rangle} ; \triangle$ vertical component $\sqrt{\left\langle w^{\prime 2}\right\rangle} ; \times$ normal-to-wall component $\sqrt{\left\langle v^{\prime 2}\right\rangle}$

side-wall. The flow is obtained in a recirculatory flume developed at the University of Trento, Italy, for the study of torrential sediment transport and debris-flow processes (Armanini et al. 2000). Shown in Fig. 14, the device features a glass-walled flume of adjustable slope (from 0 up to 23 degrees) having the following dimensions: length $=6 \mathrm{~m}$; width $=20 \mathrm{~cm}$; wall height $=40 \mathrm{~cm}$. A fast conveyer belt is used to recirculate material collected at the flume outlet. This scheme achieves steady, longitudinally uniform flow conditions within the flume.

The solid grains used for the tests are PVC particles having a cylindrical shape and the following dimensions: diameter $=3.2 \mathrm{~mm}$, height $=2.8 \mathrm{~mm}$; equivalent spherical diameter $=3.5 \mathrm{~mm}$. The volumetric concentration in a wellpacked static assembly of such grains is around $\phi_{\mathrm{rcp}} \approx 0.69$. The material density is $\rho_{s}=1,540 \mathrm{~kg} / \mathrm{m}^{3}$. Water is again used as entraining fluid. The specific flow chosen for the present testing purposes is a mature debris-flow case for which the transport layer, many grains thick, fills the entire flow depth (i.e., there is no grain-free water layer in the upper part of the flow). This case was selected because it combines a wide range of concentrations, rapid velocities, and intense shear, all desirable features to test the applicability and limits of the technique. Operating conditions for the run are: bottom slope angle $=7.2^{\circ}$; total discharge $=14.5 \mathrm{l} / \mathrm{s} ; \bar{\phi}=$ delivered solid concentration=ratio of granular discharge to water discharge $=49 \%$. The latter two parameters are derived from bulk measurements performed at the end of the run by diverting the outlet flow to a trap.

To resolve individual grains in a flowing layer of such thickness, a reasonably high image resolution is needed. For flow speeds of the order of $1 \mathrm{~m} / \mathrm{s}$, a high image acquisition frequency and an adjustable shutter are further necessary to reliably sample the grain motions and observe blur-free particles. For this purpose a high speed CCD camera was operated at a resolution of $480 \times 420$ pixels, a frequency of $250 \mathrm{f} / \mathrm{s}$, and an exposure time shuttered down to $1 / 500 \mathrm{~s}$. Because of the availability of only one camera with these characteristics, stereo viewing requires a 
a

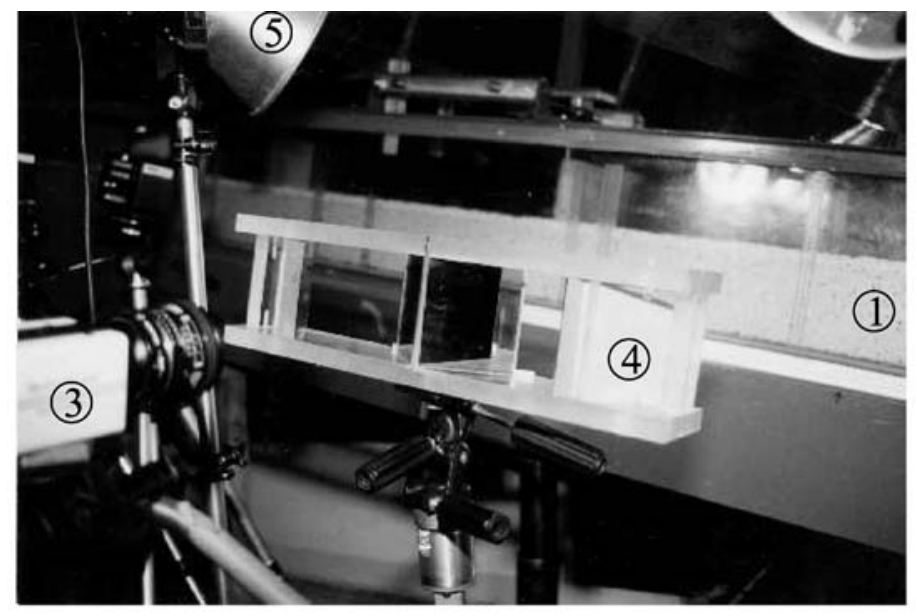

b

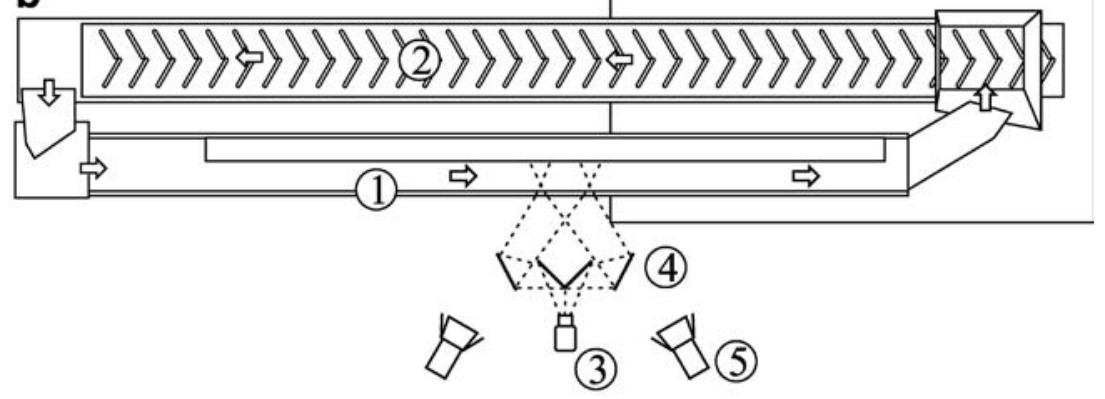

Fig. 14a, b. Recirculating flume of the Università degli Studi di Trento for the experimental study of steady uniform debris flows: a photograph; $b$ plane view; 1 flume, 2 recirculating belt, 3 camera, 4 mirror device, 5 lighting system special device composed of four mirrors arranged as shown in Fig. 14. The camera is positioned at a distance of about $1.5 \mathrm{~m}$ from the side-wall of the flume. The mirror set-up is then interposed midway between the camera and the flume.

\subsection{2}

\section{Experimental results}

Measurements obtained by application of the 3D Voronoï imaging techniques described in the present paper are shown in Figs. 15 and 16. Figure 15 displays typical trajectories of individual grains reconstituted using the stereoscopic matching and velocimetry tracking algorithms. The results shown have been filtered over three successive frames $(0.008 \mathrm{~s}$.) to suppress high-frequency noise.

Results in the $x-z$ plane show the higher velocities attained by the grains in the upper part of the flow layer. Results in the $y-z$ plane show that trajectories can be reconstituted farther away from the side-wall in the upper part of the flow. This is because concentration is lower there and occlusion effects are less severe. Despite the high concentration and the irregular nature of the granular motions, long granular trajectories are successfully reconstructed by the proposed methods.

Figure 16 shows vertical profiles for the mean velocities, fluctuation velocities, and solid concentration averaged from a sequence of 512 images. In Fig. 16a, the mean velocities in the vertical and normal to wall directions are seen to be close to zero. The mean longitudinal velocity, on the other hand, varies from zero in the static bed layer to maximum speed at the free surface. An approximately constant shear rate $\partial u / \partial z$ is obtained in the upper part of the flow. Fluctuating motions keep a roughly constant magnitude in this upper part, as shown quantitatively in Fig. $16 \mathrm{~b}$ and qualitatively in Fig. 15. There is a slight peak in the fluctuations at the free surface. This appears to be due to a corner effect involving intermittent (stick-slip) motion of partly emerged grains, interacting with the sidewall because of surface tension.

In the lowermost part, the bed is virtually motionless and fluctuations should decrease to zero. Residual values there represent noise due to inaccuracies in particle positioning. Small errors in position, uncorrelated from one
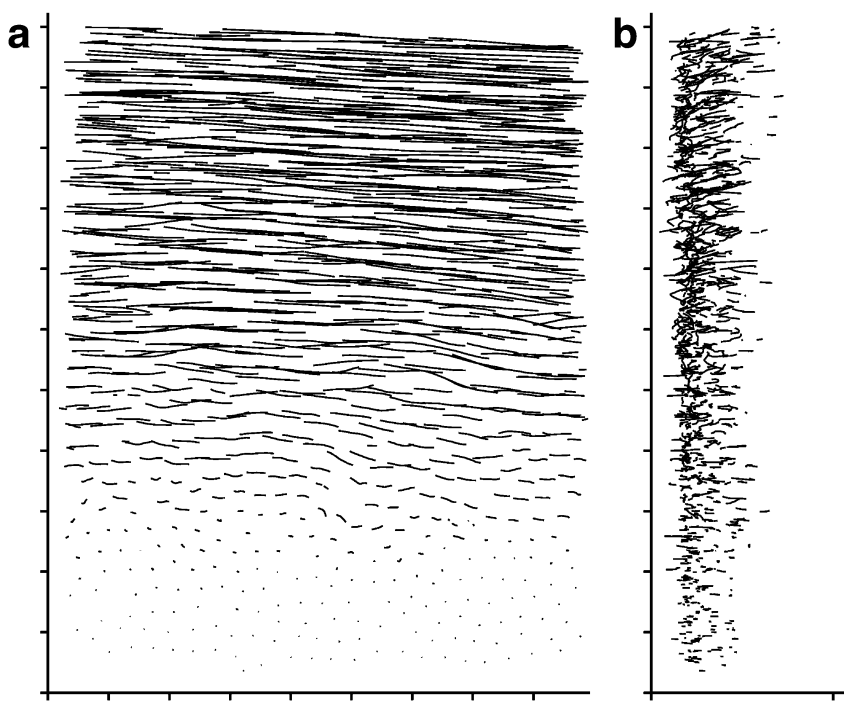

Fig. 15a, b. Granular trajectories tracked over a period of $0.04 \mathrm{~s}$ a front view, $\mathbf{b}$ "side" view obtained by projection onto the $y-z$ plane 


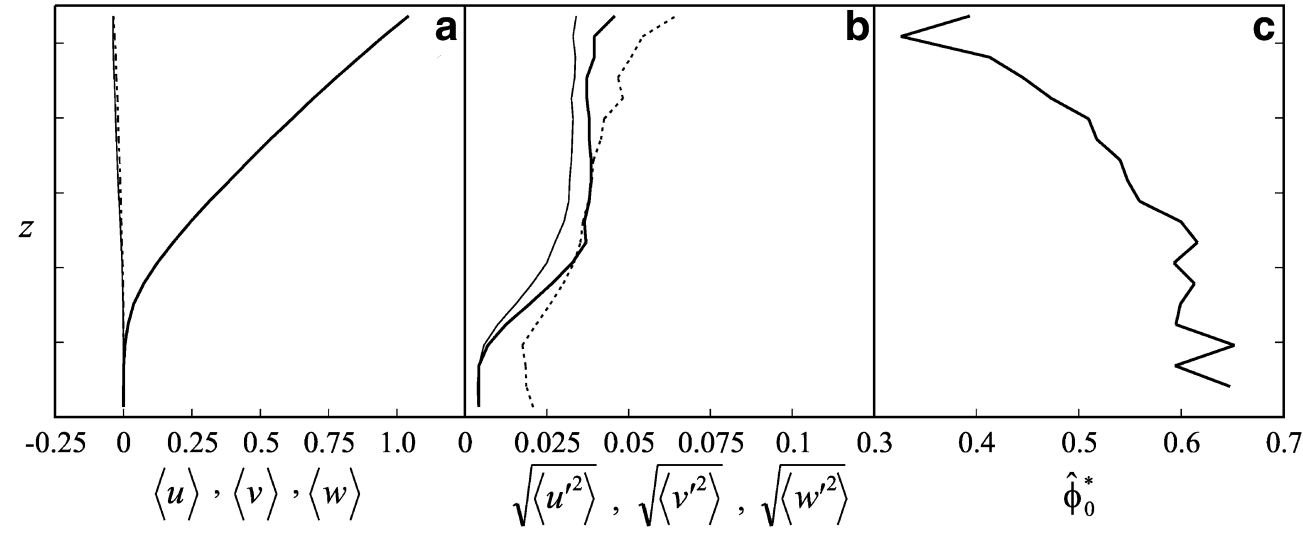

Fig. 16a-c. Vertical profiles: a mean granular velocities in $\mathrm{m} / \mathrm{s}$; b velocity fluctuations in $\mathrm{m} / \mathrm{s}$; longitudinal (bold line), vertical (solid line) and normal-to-wall (dashed line) components; c estimated granular concentration. Marks on the vertical axis represent distances of $2 \mathrm{~cm}$. The free surface of the flow is located at the top of the graphs frame to the next, translate into apparent velocity fluctuations. This effect is small for the in-plane components of the mean squared velocity fluctuations $\left\langle u^{\prime 2}\right\rangle$ and $\left\langle w^{\prime 2}\right\rangle$. Near the bed, a much higher magnitude is observed for the 5 normal to wall component $\left\langle v^{2}\right\rangle$. Not unexpectedly, this indicates that the stereo measurements of $3 \mathrm{D}$ positions achieve a lower accuracy in the depth-wise direction than in directions more closely parallel to the camera image planes. The resulting noise is seen in Fig. $16 \mathrm{~b}$ to be significant. Encouragingly, however, it does not drown out the physical signal.

Figure 16c shows the measured concentration profile obtained using adjusted estimate (33). The measured solid concentration exhibits a maximum value in the static lower layer and decreases to a minimum near the free surface. Again, corner effects perturb the measurements right at the free surface. A slightly jagged profile is observed in the lower part of the flow. This occurs because the bed is virtually motionless there: the particle configuration does not change over time hence averaging over many frames does not improve the statistics for the concentration measurements. Overall, the shape of the profile is quite regular and features reasonable concentration values. Values close to static packing are observed in the motionless bed. The following integration over depth further yields an imaging estimate for delivered concentration $\bar{\phi}$

$\bar{\phi}=\frac{\int_{z_{0}}^{z_{0}+h} \phi\langle u\rangle \mathrm{d} z}{\int_{z_{0}}^{z_{0}+h}\langle u\rangle \mathrm{d} z}$,

in which $z_{0}$ is the elevation of the rigid bed and $h$ is the flow depth. The resulting value is $\bar{\phi}=0.46$, comparable to the value of $\bar{\phi}=0.49$ derived from bulk measurements at the outlet. Even if the side-wall measurements were absolutely accurate, the two values would not be expected to perfectly coincide because the flow departs somewhat from uniformity in the transverse direction. Nonetheless, similar magnitudes should be obtained, as is the case with the present stereoscopic methods. Significantly, such agreement is obtained without any adjustment to the stereological relation derived from the fluidization tests, itself very close to the theoretically predicted relation. This was far from the case for the monocular imaging methods examined in Capart et al. (2002), for which concentration estimates had to be recalibrated in an ad hoc fashion when going from one type of experiments to another.

Conclusions

Three-dimensional imaging techniques were developed for the measurement of near-wall particulate flows. The methods include special matching and tracking algorithms, which exploit the properties of projective geometry and Voronoï diagrams to reconstruct 3D particle positions and velocities. A novel estimate based on the surface density of near-wall particles was also proposed to measure volumetric solid concentrations. Because they can handle occlusion effects and resolve position and motion ambiguities, the methods are particularly well suited for applications involving highly concentrated, rapidly sheared dispersions of many identical particles. Fluidization cell tests and an open-channel solid-liquid flow experiment were used to demonstrate the potential of the proposed techniques, as well as point out some of their limitations. It is hoped that they will prove valuable tools for the measurement of particulate flows of scientific and industrial interest.

\section{References}

Adrian RJ (1991) Particle-imaging techniques for experimental fluid mechanics. Annu Rev Fluid Mech 23:261-304

Ahuja N (1982) Dot pattern processing using Voronoï neighborhoods. IEEE PAMI 4:336-343

Armanini A, Fraccarollo L, Guarino L, Martino R, Bin Y (2000) Experimental analysis of the general features of uniform debris flow over a loose bed. In: Wieczorek GF, Naeser ND (eds) Proceedings of the 2nd International Conference on Debris-flow hazard mitigation, Taipei, August 2000. Balkema, Rotterdam, pp 327-334

Azanza E, Chevoir F, Moucheront P (1999) Experimental study of collisional granular flows down an inclined plane. J Fluid Mech 400:199-227

Bernardeau F, van de Weygaert R (1996) A new method for accurate estimation of velocity field statistics. Mon Not R Astron Soc 279:693-711

Campbell CS (1990) Rapid granular flows. Annu Rev Fluid Mech 22:57-92

Capart H (2000) Dam-break induced geomorphic flows and the transition from solid- to fluid-like behaviour across evolving interfaces. PhD thesis, Université catholique de Louvain, Belgium

Capart H, Liu HH, Van Crombrugghe X, Young DL (1997) Digital imaging characterization of the kinematics of water-sediment interaction. Water Air Soil Pollut 99:173-177

Capart H, Young DL, Zech Y (2002) Voronoï imaging methods for the measurement of granular flows. Exp Fluids 32:121-135 
Cui MM, Adrian RJ (1997) Refractive index matching and marking methods for highly concentrated solid-liquid flows. Exp Fluids 22: 261-264

De Backer A (2001) Développement et application de techniques d'imagerie digitale pour l'analyse tridimensionnelle d'un écoulement complexe. BEng thesis, Haute école Leonardo da Vinci (ECAM), Belgium (in French)

Douxchamps D, Devriendt D, Capart H, Craeye C, Macq B, Zech Y (2000) Three-dimensional reconstruction of the oscillatory freesurface of a flow over antidunes: stereoscopic and velocimetric techniques. In: Proceedings of the Oceans 2000 Conference, Rhode Island, USA, September 2000. IEEE, Piscataway, N.J.

Drake TG (1991) Granular flow: physical experiments and their implications for microstructural theories. J Fluid Mech 225:121-152

Fairhurst PG, Barigou M, Fryer PJ, Pain JP, Parker DJ (2001) Using positron emission particle tracking (PEPT) to study nearly neutrally buoyant particles in high solid fraction pipe flow. Int J Multiphase Flow 27:1881-1901

Faugeras O (1999) Three-dimensional computer vision: a geometric viewpoint. MIT Press, Cambridge, Mass.

Fortes AF, Joseph DD, Lundgren TS (1987) Nonlinear mechanics of fluidization of beds of spherical particles. J Fluid Mech 177:467-483

Guler M, Edil TB, Bosscher PJ (1999) Measurement of particle movement in granular soils using image analysis. J Comp Civ Eng 13:116-122

Hsiau SS, Jang HW (1998) Measurements of velocity fluctuations of granular materials in a shear cell. Exp Therm Fluid Sci 17:202-209

Hryciw RD, Raschke SA, Ghalib AM, Horner DA, Peters JF (1997) Video tracking for experimental validation of discrete elements simulations of large discontinuous deformations. Comput Geotech 21:235-253

Jähne B (1995) Digital image processing. Springer-Verlag, Heidelberg Berlin New York

Jain R, Kasturi R, Schunck BG (1995) Machine vision. McGraw-Hill, New York

Kang SY, Sangani AS, Tsao HK, Koch DL (1997) Rheology of dense bubble suspensions. Phys Fluids 9:1540-1561

Kim W, Kak A (1991) 3D object recognition using bipartite matching embedded in discrete relaxation. IEEE PAMI 13:224-251

Lorz U (1990) Cell-area distributions of planar sections of spatial Voronoï mosaics. Mater Characteriz 25:297-309

Malik NA, Dracos T, Papantoniou D (1993) Particle tracking velocimetry in three dimensional flows - II: Particle tracking. Exp Fluids 15:279-294

Menon N, Durian DJ (1997) Diffusing-wave spectroscopy of dynamics in a three-dimensional granular flow. Science 275:1920-1922

Murai Y, Matsumoto Y, Yamamoto F (2001) Three-dimensional measurement of void fraction in a bubble plume using statistic stereoscopic image processing. Exp. Fluids 30:11-21

Nakagawa M, Altobelli SA, Caprihan A, Fukushima E, Jeong EK (1993) Noninvasive measurements of granular flows by magneticresonance-imaging. Exp Fluids 16:54-60
Natarajan VVR, Hunt ML, Taylor ED (1995) Local measurements of velocity fluctuations and diffusion coefficients for a granular material flow. J Fluid Mech 304:1-25

Okabe A, Boots B, Sugihara K (1992) Spatial tessellations: concepts and applications of Voronoï diagrams. Wiley, Chichester

Phillips RJ, Armstrong RC, Brown RA (1992) A constitutive equation for concentrated suspensions that accounts for shear-induced particle migration. Phys Fluids A 4:30-40

Preparata FP, Shamos MI (1985) Computational geometry: an introduction. Springer-Verlag, Heidelberg Berlin New York

Richardson JF, Zaki WN (1954) Sedimentation and fluidization: part I. Trans Instn Chem Engrs 32:35-53

Ross SM (1990) A course in simulation. Macmillan, Basingstoke

Rouyer F, Lhuillier D, Martin J, Salin D (2000) Structure, density, and velocity fluctuations in quasi-two-dimensional non-Brownian suspensions of spheres. Phys Fluids 12:958-963

Savage SB, Dai R (1993) Studies of granular shear flows, wall slip velocities, "layering" and self-diffusion. Mech Mater 16:225238

Seymour JD, Caprihan A, Altobelli SA, Fukushima E (2000) Pulsed gradient spin echo nuclear magnetic resonance imaging of diffusion in granular flow. Phys Rev Lett 84:266-269

Song XQ, Yamamoto F, Iguchi M, Murai Y (1999) A new tracking algorithm of PIV and removal of spurious vectors using Delaunay tessellation. Exp Fluids 26:371-380

Spinewine B, Capart H, Zech Y (2000) Stereoscopic imaging measurement of solid concentrations in dense granular flows. In: Proceeding of the 10th International Conference on Transport and sedimentation of solid particles, Wroclaw, September 2000. Akademia Rolnicza we Wroclawiu, Wroclaw, Poland, pp 497-509

Trucco E, Verri A (1998) Introductory techniques for 3-D computer vision. Prentice Hall, Upper Saddle River, N.J.

Tsai RY (1987) A versatile camera calibration technique for highaccuracy 3D machine vision metrology using off-the-shelf TV cameras and lenses. IEEE J Robotics Auto 3:323-344

Ushijima S, Tanaka N (1996) Three-dimensional particle tracking velocimetry with laser-light sheet scannings. J Fluid Eng 118:352357

Veber P, Dahl J, Hermansson R (1997) Study of the phenomena affecting the accuracy of a video-based particle tracking velocimetry technique. Exp Fluids 22:482-488

Virant M, Dracos T (1997) 3D PTV and its application on Lagrangian motion. Meas Sci Technol 8:1539-1552

Wildman RD, Huntley JM, Parker DJ (2001) Granular temperature profiles in three-dimensional vibrofluidized granular beds. Phys Rev E 63:1311-1320

Wildman RD, Huntley JM, Hansen JP (1999) Self-diffusion of grains in a two-dimensional vibrofluidized bed. Phys Rev E 60:7066-7075

Zhang DZ, Prosperetti A (1994) Averaged equations for inviscid disperse two-phase flow. J Fluid Mech 267:185-219 